\title{
Adhesive force model at a rough interface in the presence of thin water films: the role of relative humidity
}

\author{
M. Bazrafshan ${ }^{1,2 *}$, M.B. de Rooij ${ }^{2}$, D.J. Schipper ${ }^{2}$ \\ ${ }^{1}$ Materials innovation institute (M2i), Elektronicaweg 25, 2628 XG , Delft, Netherlands \\ ${ }^{2}$ Laboratory for Surface Technology and Tribology, Department of Engineering Technology, \\ University of Twente, P.O. box 217, 7500AE, Enschede, Netherlands
}

\begin{abstract}
:
This paper proposes a Boundary Element Model (BEM) for the adhesive contact at the rough interface of two contacting bodies, where a thin water film is adsorbed on the surfaces due to the condensation from the humid environment. Three adhesive components contribute to the total adhesive force: solid-solid and liquid-solid van der Waals interactions and capillary force. Rather than a film with uniform thickness, the true distribution of the water film over the surfaces is considered. The capillary component of the adhesive force is first verified through the wellknown capillary force model at the smooth contact of a rigid ball-on-flat configuration for different values of the Relative Humidity $(R H)$ of the environment. Then, the adhesive contact at a rough interface with three different relative auto-correlation lengths under different normal loads is considered. It is found that the capillary force dominates the total adhesive force and it increases with $R H$, while the other two adhesive components are rather constant. In addition, the capillary force appears to first increase with $\mathrm{RH}$ and then decrease as almost the entire noncontact area of the interface is covered by a meniscus. This variation in the capillary force depends on the auto-correlation length, roughness rms of the rough interface, and the normal
\end{abstract}


load. Furthermore, it is confirmed that the capillary force, while employing a water film with uniform thickness, deviates that of the true distribution of this film.

\section{Keywords: Adhesion, Capillary, Thin Water Film, Boundary Element Method}

\section{Nomenclature}

\begin{tabular}{|c|c|c|c|}
\hline A & area of target domain & $R H$ & relative humidity \\
\hline$a$ & contact radius & $r_{1,2}$ & meniscus radii \\
\hline$E^{*}$ & reduced elastic modulus & $r_{k}$ & Kelvin radius \\
\hline$e$ & film thickness & $S$ & equivalent meniscus height \\
\hline$e_{a}$ & air gap & $s_{m}$ & meniscus height \\
\hline$F_{0}$ & external normal force & $T$ & temperature \\
\hline$F_{a d h}$ & adhesion force & $t_{1,2}$ & film thickness on bodies 1 and 2 \\
\hline$F_{\text {cap }}$ & capillary force & V & water molar volume \\
\hline$F_{e l}$ & electro-static force & $V_{a d s}$ & adsorbed water volume \\
\hline$F_{\text {total }}$ & total adhesive force & $z_{0}$ & equilibrium distance \\
\hline$F_{v d W}$ & van der Waals adhesive force & $\Delta P_{\text {cap }}$ & capillary pressure \\
\hline$g$ & local gap & $\Delta \gamma$ & work of adhesion \\
\hline$h_{0}$ & maximum allowable gap for adhesion & $\Omega$ & computation domain \\
\hline$h_{s}$ & roughness height & $\Omega_{\text {wetted }}$ & meniscus-wetted areas \\
\hline
\end{tabular}




$\begin{array}{llll}h_{w} & \text { water film free surface } & \Omega_{\text {ws }}^{1} & \text { water-solid interaction area, } \\ & & \text { scenario } 1 \\ L & \text { size of the target domain } & \Omega_{\text {ws }}^{2} & \text { water-solid interaction area, } \\ & & & \text { scenario } 2 \\ L_{a c} & \text { auto-correlation length } & \gamma & \text { surface tension } \\ N & \text { no. of grid points in each direction } & \eta & \text { viscosity } \\ p & \text { pressure } & \theta_{1,2} & \text { contact angles of bodies } 1 \text { and } 2 \\ p_{s s} & \text { solid-solid adhesive pressure } & \sigma & \text { roughness rms } \\ R & \text { ball radius } & & \\ R_{g} & \text { global gas constant } & \sigma_{0} & \text { Dugdale stress }\end{array}$

\section{Introduction}

Along with the rapid development of micro-nano electromechanical systems (MEMs/NEMs), intermolecular adhesive interactions between two surfaces has attracted a lot of attention among the researchers. In such devices, adhesion is a common failure mechanism and a major reliability concern since strong adhesive forces arise due to the high surface energies of the contacting solids and or the formation of liquid menisci between contacting surfaces leading to undesirable friction forces. Prediction of these adhesive forces is crucial for the design of micro/nano devices. To reach this purpose, providing analytical models is, although desirable, almost impossible due to the complexity of the geometry of the contacting surfaces. Thus, numerical modeling is a suitable alternative for such cases. 
Bradley was the first in studying adhesion in contact mechanics by looking at the adhesive contact between two rigid spheres [1]. Then, two opposing models, JKR [2] and DMT [3], were proposed for spherical elastic contacts. It was shown by Tabor that, regardless of their different approaches and assumptions, both models were correct as they were two opposite extremes of a single theory. The JKR model is valid for the case of large and compliant contacts. The DMT model, whereas, is suitable for the small and stiff contacts. Following these two models, Maugis proposed an adhesive model (known as Maugis-Dugdale (MD) model) for this spherical contact through a Dugdale approximation of the Lennard-Jones potential (which defines the adhesive energy) [4].

Although such analytical models provide exact solution to the adhesive problem, they are limited to smooth contacts with simple shapes. For the adhesive contact of rough surfaces, however, numerical methods are essential. Several authors have attempted to numerically evaluate the adhesion between two rough surfaces through multi-asperity [5,6], finite element $[7,8]$, statistical [9-11], molecular dynamics [12-14], and boundary element models (BEM) [15,16]. Yet, there is a lot of discussion in the scientific community in this regard [17-22]. Vakis et al recently conducted a very comprehensive review of these methods along with the tribological applications in different scales [23].

The strong adhesion bond between the sand particles in a sandcastle, powders and sand in granular materials, and so forth is due to the liquid menisci forming around the contact area of two adjacent particles. The corresponding force caused by this phenomenon is called meniscus or capillary force. Understanding this phenomenon is important in granular materials, adhesion of insects, geckos, and spiders, and micro-nano electromechanical systems. Haines [24] and then Fisher [25] were among the first who realized the importance of capillary forces. 
Investigation of the meniscus formation between smooth surfaces of different configurations such as sphere-sphere, sphere-plane, plane-plane, cone-cone, cone-plane and so on can be found frequently in literature [26-32]. The transition of water from ordered to bulk form as a function of the relative humidity has been investigated for a ball-on-flat configuration in [33-35]. It was shown that for low values of relative humidity ( $R H<30 \%$ ), 1 to 3 monolayers of water molecules are adsorbed on the surface behaving like ice and therefore, no meniscus forms. Consequently, the adhesive force is only contributed by the van der Waals force due to the solisolid and liquid-solid interactions. At higher levels of relative humidity, however, the capillary force is responsible for the adhesive force.

To study the meniscus formation and capillary force between rough surfaces, researchers have pursued different numerical approaches such as multi-asperity, statistical, and boundary element approaches. Xue and Polycarpou coupled a single asperity meniscus model, based on the extended Maugis-Dugdale elastic theory, with a statistical roughness surface and presented an improved multi-asperity meniscus force model for rough contacts [36]. de Boer proposed a multi-asperity model by extending the Greenwood-Williamson model of rough surfaces [37]. Wang and Regnier developed a capillary adhesion model for the contact of a single asperity of a power law shape with a flat surface and extended it to the contact between rough surfaces [38]. Based on the fractal theory, describing the behavior of multiple roughness scales and the Gaussian roughness distribution, You and Wan proposed a model to account for the van der Waals and capillary forces between a rough particle and surface [39]. Peng et al studied the capillary adhesion of rough fractal surfaces [40]. They implemented a Dugdale approximation of the Laplace pressure to express adhesive interaction at the contact interface. As the main 
limitations of such multi-asperity models, one should note that merely the summits are considered and the rest of the surface is discarded.

Tian and Bhushan proposed a numerical algorithm to study the micro-meniscus effect of a very thin liquid film on the static friction of rough surface contacts [41]. They modified the classical meniscus theory of a single-sphere contact to include the effect of multi-asperity contacts in the presence of an ultra-thin liquid film adsorbed on the contacting surfaces. In their calculation, however, they did not incorporate the elastic deformations due to the adhesive pressure. Lin and Chen developed a model for the adhesive meniscus force at the interface of a rough surface and a smooth surface covered by a thin water film [42]. They also considered the variation of the water film as a normal load is applied. Rostami and Streator proposed a deterministic approach to study the liquid-mediated adhesion between rough surfaces [43]. Based on the liquid volume available at the interface, they defined a wetting radius and the non-contact areas inside the wetting radius would experience a constant capillary pressure. They, however, neglected the contribution of mobile liquid at the interface, which indeed contributes to the meniscus formation. Hence, a comprehensive model to predict the adhesive force between two rough surfaces in the presence of the adsorbed liquid films is still in need.

In this paper, we present a boundary element model to analyze the adhesive contact between two rough surfaces where thin films of water are adsorbed on the contacting surfaces mediating the formation of meniscus. The total volume of adsorbed water depends on the contacting materials and increases with relative humidity. The thickness of the water films varies over the interface in such a way to fulfil the mechanical equilibrium and Newtonian rheology of water. Three components contribute to the total adhesive force: solid-solid and water-solid van der Waals interactions and capillary force. The deformation due to the adhesive pressure is also included in 
the total deformation of the interface. The next section introduces these components and formulates their contribution to the total adhesive force.

\section{Theory and modeling}

When two surfaces are brought into contact under an external normal load, an attractive force pulls the two surfaces together. This adhesive force might originate from different sources: van der Waals interactions between the opposing molecules on the two surfaces, a capillary force due to the meniscus pressure, an electrostatic force due to a possible electric charge on the surfaces, and so forth. The total work of adhesion is the work required to put into the system to separate these two surfaces from equilibrium to an infinite distance. In general, the total adhesive force is the summation of all these attractive forces:

$$
F_{a d h}=F_{v d W}+F_{c a p}+F_{e l}+\ldots
$$

In this paper, we study the van der Waals and capillary forces as the main contributors to the adhesive force between two rough surfaces. The total van der Waals force comes from two types of interaction: solid-solid and water-solid interaction. In the following, these two interactions as well as the capillary force are modeled.

\section{1. van der Waals forces}

The van der Waals forces are distant dependent interactions between atoms which are divided into three categories: dipole-dipole interactions, randomly induced dipole interactions, and 
interaction between non-polar molecules [44]. The adhesive pressure, $p_{s s}$, due to van der Waals interactions is expressed through the Lennard-Jones potential (neglecting any capillary or electrostatic forces) as an explicit function of the separation, $g$ :

$$
p_{s s}=\frac{8 \Delta \gamma}{3 z_{0}}\left(\left(\frac{z_{0}}{g+z_{0}}\right)^{9}-\left(\frac{z_{0}}{g+z_{0}}\right)^{3}\right)
$$

where $\Delta \gamma$ is the work of adhesion due to solid-solid van der Waals interaction (see Fig. 1). In the equation above, $z_{0}$ is the equilibrium separation, typically ranging from $0.2 \mathrm{~nm}$ to $0.4 \mathrm{~nm}$.

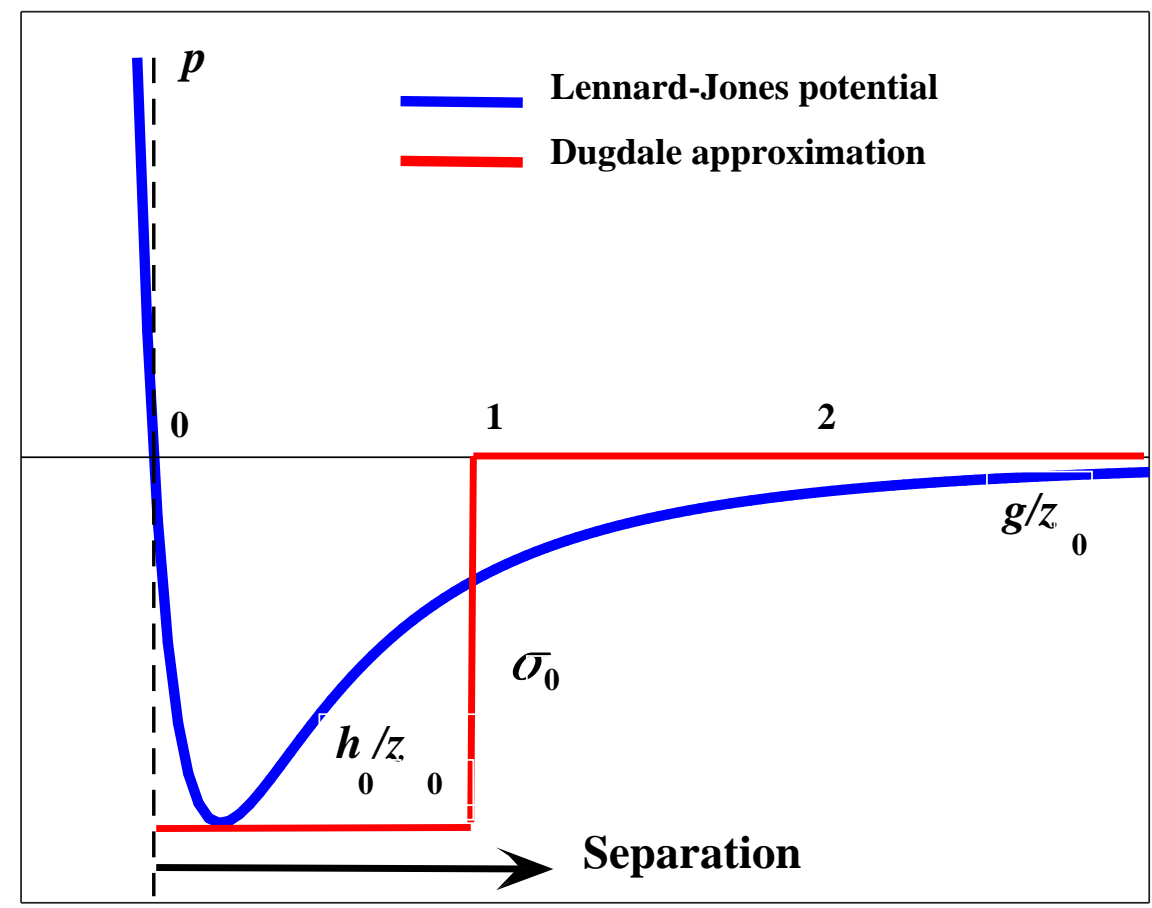

Fig. 1. Dugdale approximation of Lennard-Jones potential (For interpretation of the references to color in this figure legend, the reader is referred to the web version of this article). 
Bazrafshan et al proposed a CGM (Conjugate Gradient Method) based BEM algorithm by means of a Dugdale approximation for the total work of adhesion to solve the adhesive normal contact between two bodies, given by [16]:

$$
\begin{array}{lccc}
p>-\sigma_{0} & \text { at } & g=0 & \text { (a) } \\
p=-\sigma_{0} & \text { at } & 0<g<h_{0} & \text { (b) } \\
p=0 & \text { at } & g>h_{0} & \text { (c) } \\
\int_{\Omega} p d x d y=F_{0} & & & \text { (d) }
\end{array}
$$

in which, $-\sigma_{0}$, the maximum attractive pressure of the Lennard-Jones potential, is applied such that [4]:

$$
\Delta \gamma=\sigma_{0} h_{0}, \quad \sigma_{0}=\frac{16 \Delta \gamma}{9 \sqrt{3} z_{0}}
$$

This results in $h_{0}=9 \sqrt{3} z_{0} / 16=0.974 z_{0}$. The parameter $h_{0}$ is the maximum allowable gap for the presence of adhesion (the constant negative stress $-\sigma_{0}$ as a Dugdale approximation of adhesion energy). In other words, in non-contact regions, a constant negative stress $\left(-\sigma_{0}\right)$ is present as long as the local separation is smaller than $h_{0}$.

\subsection{Capillary force}

Liquids with small contact angles spontaneously condense from vapor to fill in cracks and pores. At a dry and smooth ball-on-flat interface, water condenses to form a meniscus outside the contact area (Fig. 2). At thermodynamic equilibrium, the meniscus radius of curvature or namely Kelvin radius, $r_{k}$, is directly controlled by the relative humidity ( $R H$ ) as [44]: 


$$
\left(\frac{1}{r_{1}}-\frac{1}{r_{2}}\right)^{-1}=r_{k}=\frac{\gamma V}{R_{g} T \log R H}
$$

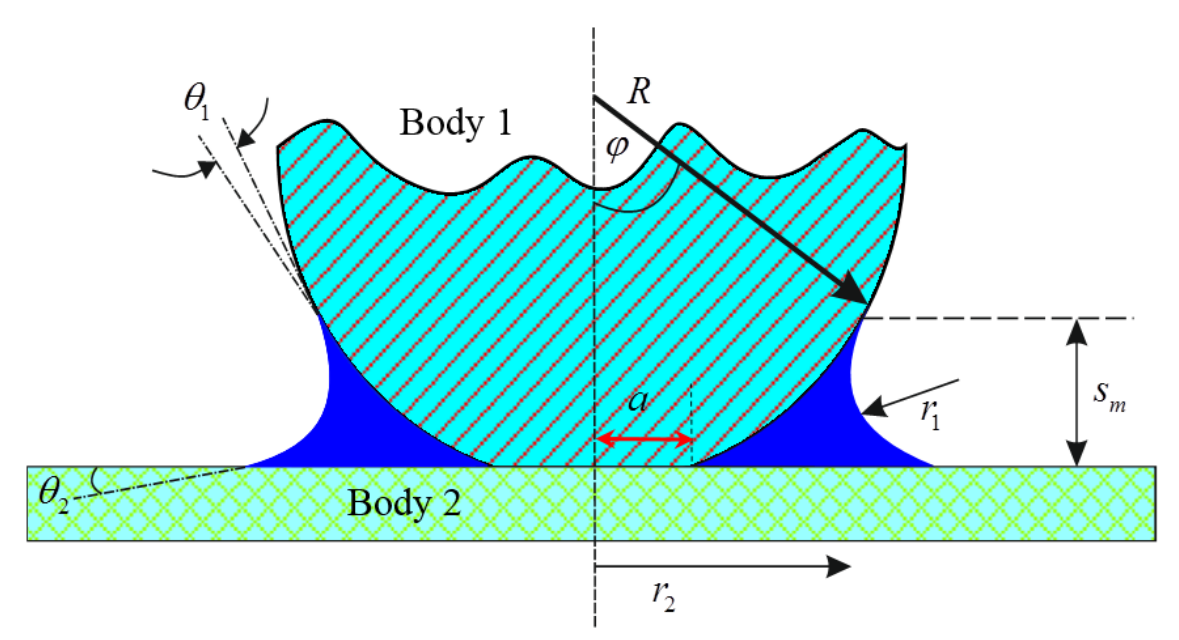

Fig. 2. Meniscus formation at a smooth ball-on-flat interface.

where $1 / r_{1}$ and $1 / r_{2}$ are the meniscus curvatures, $V$ the molar volume of water, $R_{g}$ the universal gas constant, and $T$ the absolute temperature.

When two rough surfaces are brought into contact under a light normal load, only a few pairs of asperities come into contact. In the presence of a thin adsorbed water film, a meniscus forms around the contacting and near-contacting asperities [44]. The pressure inside the meniscus is smaller than that outside the meniscus, resulting in an additional pulling force acting on the contacting surfaces.

\subsubsection{Leveling of a thin water film over a rough surface}

A thin film of water over a rough surface is neither flat nor the form of the rough surface, but follows the form illustrated in Fig. 3. The uneven distribution of this film over a rough surface is 
due to Laplace-Young pressure difference at summits and valleys of the surface. Having the topography of the surface, $h_{s}(x, y)$, and the volume of adsorbed water on it, $V_{\text {ads }}(R H)$, one can find the true uneven distribution by numerically solving the equations of mechanical equilibrium and rheology of the water. To do this, suppose that the water film is deposited on the surface at $t=0$ and the initial thickness, $e(x, y, 0)$, is assumed to be uniform all over the surface, computed as:

$$
e(x, y, 0)=\frac{V_{a d s}(R H)}{A}
$$

The water film is then non-uniformly distributed on the surface as time passes to reach a stable level while the volume of water is constant, and the same as its initial value, at any time as:

$$
\int_{\Omega} e(x, y, t) d x d y=V_{a d s}(R H)
$$

Combining the mechanical equilibrium and Newtonian rheology for water and neglecting the gravity reads [45]:

$$
\begin{aligned}
\frac{\partial h_{w}}{\partial t}= & -\frac{\gamma}{3 \eta} \frac{\partial}{\partial x}\left[\left(h_{w}-h_{s}\right)^{3}\left(\frac{\partial^{3} h_{w}}{\partial x^{3}}+\frac{\partial^{3} h_{w}}{\partial x \partial y^{2}}\right)\right] \\
& -\frac{\gamma}{3 \eta} \frac{\partial}{\partial y}\left[\left(h_{w}-h_{s}\right)^{3}\left(\frac{\partial^{3} h_{w}}{\partial y^{3}}+\frac{\partial^{3} h_{w}}{\partial y \partial x^{2}}\right)\right],
\end{aligned}
$$

where $h_{w}(x, y, t)=e(x, y, t)+h_{s}(x, y)$ is the height of the water free surface. When the water free surface reaches a stable level, it does not change with time. Eq. (7) is a set of high order nonlinear partial differential equations which is numerically solved using the method of lines. If the input rough profile, $h_{s}$, has $N^{2}$ surface elements (and so does $h_{w}$ ), the method of lines converts 
Eq. (7) to a set of $N^{2}$ coupled ordinary differential equations of the first order. Since the roughness is assumed to be periodic, the derivation property of the Fourier Transform can be exploited to convert the derivatives to the function nodal values. Then, this set of $N^{2}$ ordinary differential equations are solved using the adaptive step-size Runge-Kutta scheme [45].

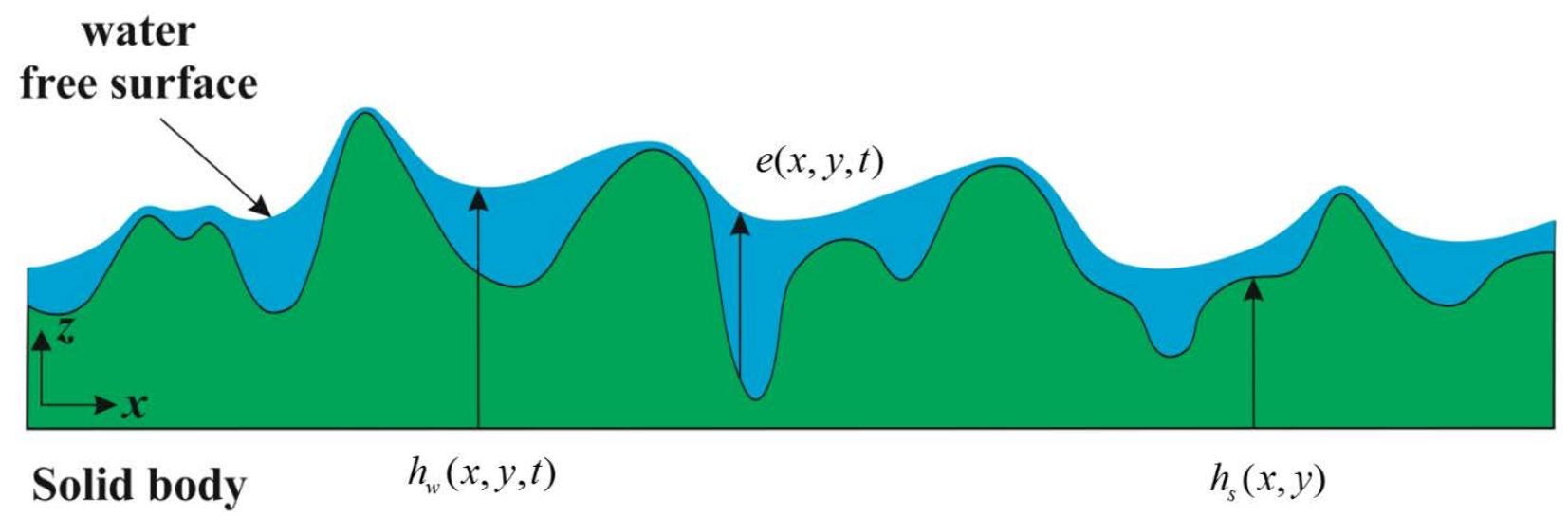

Fig. 3. Leveling of a water film over a rough surface.

\subsubsection{Meniscus formation between two rough surfaces}

When the two surfaces approach one another, several isolated micro-menisci occur at the contact interface around the contacting asperities and also at near-contacting asperities (Fig. 4(a)). This formation is dependent on the relative humidity and roughness parameters.

It is first assumed in this study that there is no water flow between the contacting surfaces. We also neglect the squeezed water when two counter asperities come into contact. This assumption underestimates the capillary force since this squeezed water can help to form larger menisci and thus, larger capillary force. This is even more significant for surfaces with long auto-correlation lengths as the available water film at the peaks is comparatively thicker due to smaller local gradients. For the sake of simplicity, however, we neglect the effect of this squeezed film. There 
are two mechanisms governing the formation of meniscus around an asperity contact. One is the condensation from the humid environment and the other one is surface migration-controlled growth [41]. These two mechanisms are distinguished by the source from which water required for the meniscus formation is supplied. Either or both of these mechanisms could be involved in the meniscus formation process. It is here assumed that water is supplied through capillary condensation from the humid environment to form the meniscus. This way, the original volume of adsorbed water is kept the same after the contact. As a result, the distribution of the thin water films does not change as the surfaces come into contact at a few asperities; at least it can be claimed that the film thickness changes much more in the valleys than in the summits (which is out of interest as the valleys have a lower contribution to the total adhesion). Therefore, one can keep the original distribution of water films (before the contact) for the calculations in the next section.

The total capillary force is a function of the meniscus shape and volume. Since determining the exact shape of meniscus is demanding, we conduct a simplified approach by approximating the effective section of a meniscus as an arc so that the meniscus height is given by [41]:

$$
s_{m}=r_{k}\left(\cos \theta_{1}+\cos \theta_{2}\right)
$$

Here, $\theta_{1}$ and $\theta_{2}$ are the contact angles (see Fig. 2). It must be noted that, in the presence of water films, the curvature of the meniscus does not significantly change the thickness of the adsorbed film [46]. Therefore, the meniscus rise to $2 r_{k}$ rather than the one expressed in Eq. (8). We also neglect the variation of the meniscus height around the irregular asperities.

At this stage, it is required to locate the areas wetted by meniscus. These areas experience the capillary pressure. To do this, it is easier to first combine the heights of the two rough surfaces 
and put the summation of the thickness of the two water films on a smooth surface as shown in Fig. 4(b). It is first important to mention that the meniscus-wetted areas are the ones whose local gap from the counter surface is smaller than the summation of the local thickness of the water films. The cross-cut areas of the combined roughness at the meniscus height of $S=2 r_{k}+e_{1}+e_{2}$ ( $e_{1}$ and $e_{2}$ are the local thickness of the water films on bodies 1 and 2 as obtained through the leveling procedure described in section 2.2.1) which are linked to the meniscus-wetted areas are considered to experience the capillary pressure. In other words, the cross-cut area at the meniscus height is first grouped into individual islands. Then, those islands, which overlap the meniscuswetted areas, are selected to undergo the capillary pressure and the rest of the islands are discarded (see Fig. 4(c)).

Thermal fluctuations at molecular level can roughen the free surface of water films [47]. In addition, the van der Waals interaction between water films of the opposite sides at locations where the two films are very close to one another needs to be taken into account (see the small gap prone to form a meniscus in Fig. 4(a)). Combining these two effects at such locations, the two films can jump into contact and quickly form a meniscus similar to the one around the nearcontacting asperities. To treat this behavior, we define a critical distance, $d_{c r}$, to distinguish whether such a meniscus forms or not. There is no strict criterion for the value of this parameter. However, it is expected to be in the order of 1-3 diameters of a water molecule. Here, we assume this parameter to be three times the size of a water molecule.

To put it all together, micro-menisci islands can form in three types of places at the interface while meeting either of the mentioned criteria: around the contacting asperities, at near 
contacting asperities, and at areas very small gap (smaller than $d_{c r}$ ) prone to form a micro-

meniscus. In all these wetted areas, $\Omega_{\text {wetted }}$, a capillary pressure of $\Delta P_{\text {cap }}$ exists:

$$
\Delta P_{\text {cap }}=\frac{\gamma}{r_{k}}=\frac{V}{R_{g} T \log R H}
$$

\section{(a)}

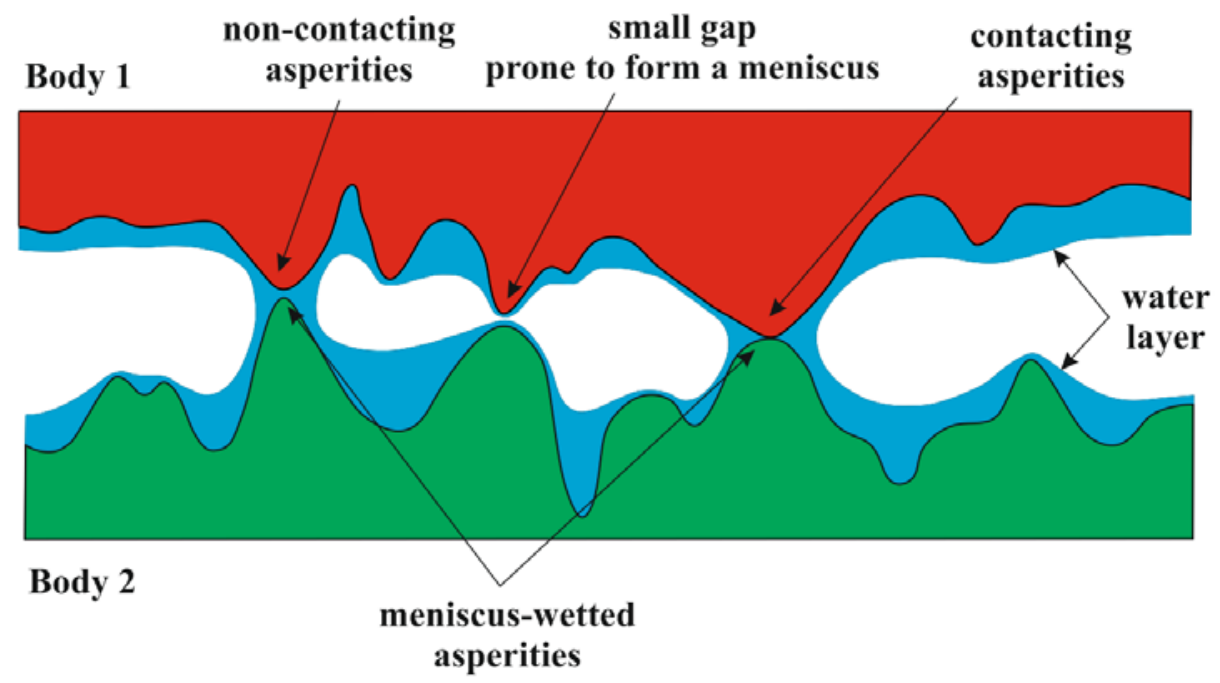

(b)

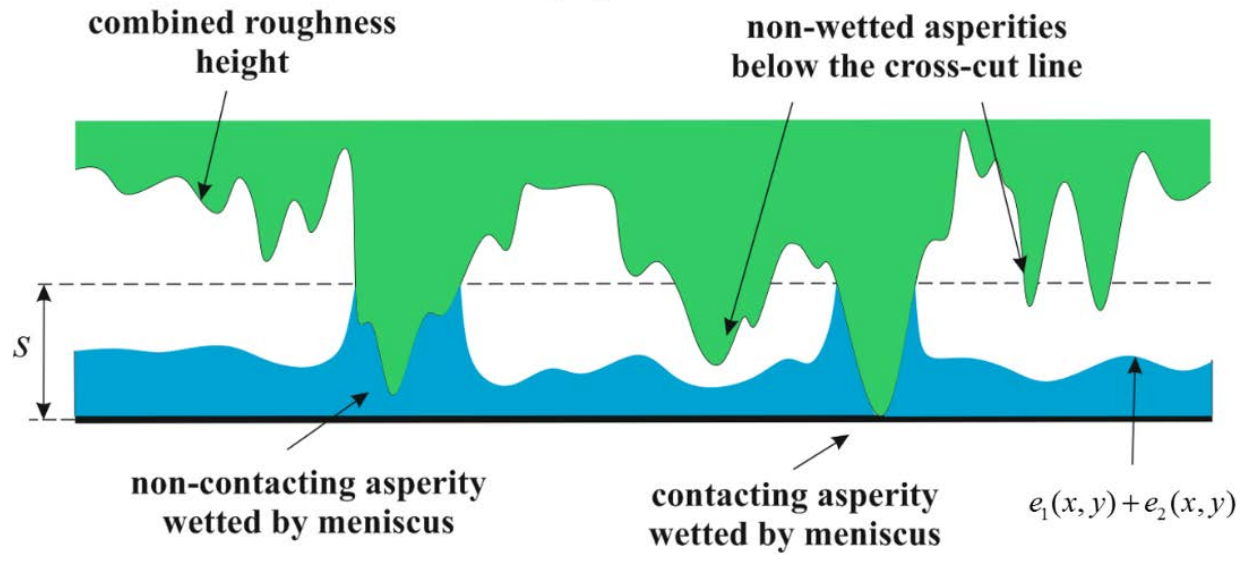




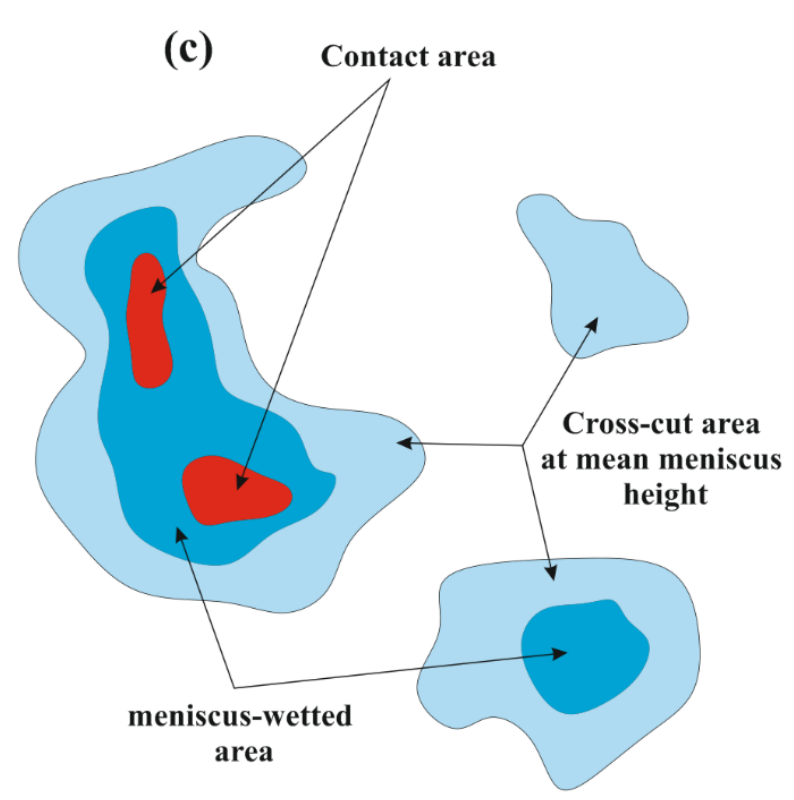

Fig. 4. (a) Meniscus formation at the contact of two rough surfaces in the presence of adsorbed water films (b) strategy to find meniscus-wetted asperities (c) a schematic diagram of contact area, meniscus-wetted area, and crosscut area at mean meniscus height.

\subsection{Water-Solid interaction}

The other component in the adhesive force, which needs to be taken into consideration, is the interaction between the two solids through water and air (or vacuum) as media. In this case, two scenarios exist depending of the local gap between each two counter elements. The first scenario takes place where the local gap between these two nodes is smaller than the summation of the local thickness of the two water films (Fig. 5(a)). Therefore, the thickness of the trapped water, $e_{w}$, is the same as the local gap and the local adhesive stress is given by [44]:

$$
P_{w s}^{1}=-\frac{A_{1 w 2}}{6 \pi e_{w}^{3}}
$$


where $A_{1 w 2}$ is the Hamaker constant for media 1 and 2 interaction across water. The region at the interface eligible for this scenario is named $\Omega_{w s}^{1}$.

(a)

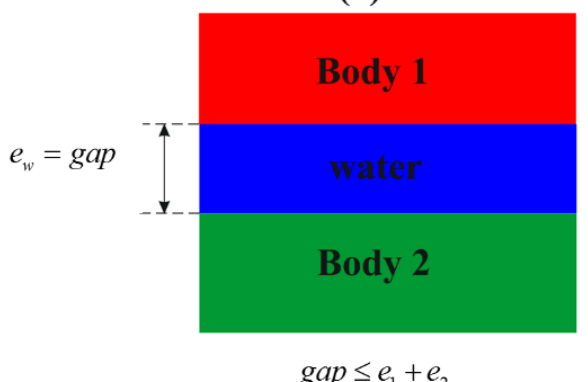

$g a p \leq e_{1}+e_{2}$ (b)

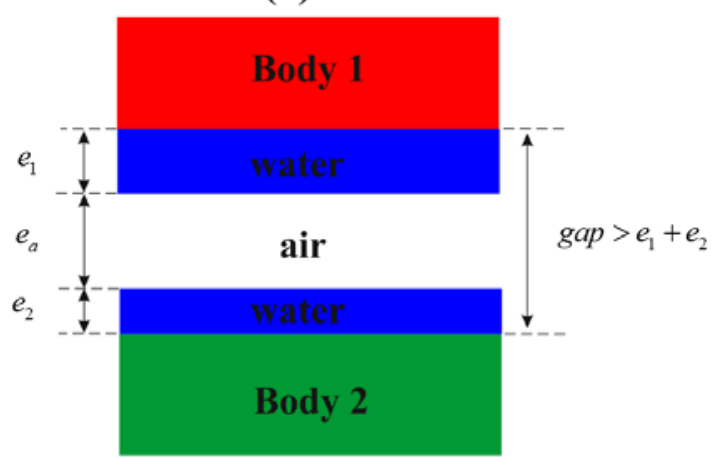

Fig. 5. Two different scenarios for the water-solid interaction.

The second scenario occurs where the local gap is larger than the summation of the local thickness of the two water films (Fig. 5(b)). The region at the interface eligible for this scenario is named $\Omega_{w s}^{2}$. For this case, the local stress is expressed as [44]:

$$
P_{w s}^{2}=-\frac{1}{6 \pi}\left(\frac{A_{\text {waw }}}{e_{a}^{3}}-\frac{\sqrt{A_{1 w 1} A_{\text {waw }}}}{\left(e_{a}+e_{1}\right)^{3}}-\frac{\sqrt{A_{2 w 2} A_{\text {waw }}}}{\left(e_{a}+e_{2}\right)^{3}}+\frac{\sqrt{A_{1 w 1} A_{2 w 2}}}{\left(e_{a}+e_{1}+e_{2}\right)^{3}}\right)
$$

where $A_{i k j}$ is the Hamaker constant for media $i$ and $j$ interacting across medium $k$ and $a$ and $w$ stand for air and water, respectively.

One needs to note that the largest and dominating term in Eq. (11) is $A_{\text {waw }} / e_{a}^{3}$ since the denominators of the other terms are comparatively much greater than $e_{a}$. On the other hand, considering the fact that $e_{a}$ has to be larger than $d_{c r}$, the third power of $e_{a}$ makes it very small 
compared to the case of Eq. (10). Therefore, it can be concluded that the water-solid interaction

\section{is dominated by scenario (a) in Fig. 5.}

\section{Numerical algorithm}

This section summarizes the numerical scheme of the proposed approach. The present contact problem is the extension of Eq. (3) (which defines the adhesive contact problem in the presence of only van der Waals forces) to include the capillary pressure and van der Waals water-solid interaction described in sections 2.2 and 2.3. This equation suggests that there is no pressure at areas where separation is greater than $h_{0}$. In presence of liquid films and capillary effect, however, such areas experience either or both of the capillary force and water-solid van der Waals interaction. Therefore, the present contact problem is given by:

$$
\begin{array}{lrcr}
p>-\sigma_{0} & \text { at } & g=0 & (a) \\
p=-\sigma_{0} & \text { at } & 0<g<h_{0} & (b) \\
p=\Delta P_{c a p} & \text { at } & \Omega_{\text {wetted }} & (c) \\
p=P_{w s}^{1} & \text { at } & \Omega_{w s}^{1} & (d) \\
p=P_{w s}^{2} & \text { at } & \Omega_{w s}^{2} & (e) \\
\int_{\Omega} p d x d y=F_{0} & & & (f)
\end{array}
$$

It is noted that for the solid-solid van der Waals interaction to be present, the local gap is required to be smaller than $h_{0}$ which is smaller than (or perhaps almost equal to) the size of a water molecule. In such areas, thus, no meniscus or water-solid interaction can exist. In order to solve the contact problem of Eq. (12) for the unknown pressure, $p$, a BEM-based approach is implemented. The boundary element method (BEM) is a numerical method which 
transforms governing partial differential equations into integral equations over the surface or boundary of a domain [48]. Therefore, rather than discretizing the entire 3D domain, the boundary (here the contact interface) is divided into small patches where the unknown functions are approximated in terms of nodal values, and the integral equations are discretized and solved numerically. As all the approximations are transformed to the boundary, the BEM has better accuracy and efficiency (since the dimensionality reduces by one order) in contact of rough surfaces than other numerical methods such as finite element method and molecular dynamics.

Fig. 6 depicts the flowchart of the proposed numerical algorithm. In the first step, mechanical and surface properties of the contacting bodies as well as the loading conditions, surface topographies, and relative humidity are input to the algorithm. The relative humidity, along with the adsorption properties of the contacting surfaces, is used to estimate the volume of adsorbed water on the surfaces in a humid environment [49]. An alternative to this is to measure this parameter at different values of the relative humidity of the environment which gives out an adsorption isotherm. Here, the volume of adsorbed water is used to calculate the distribution of the thin water films on the surfaces as explained in section 2.2.1.

In step 2, an initial guess is provided for the pressure. Using this initial pressure, in step 3, the corresponding deformation at the interface is computed as:

$$
u(x, y)=\int_{\Omega} k(x-\zeta, y-\eta) p(\zeta, \eta) \mathrm{d} \zeta \mathrm{d} \eta
$$

where $x$ and $y$ are the spatial coordinates and $k(x, y)$ is the Boussinesq kernel function and is expressed as [50]: 


$$
k(x, y)=\frac{1}{\pi E^{*}} \frac{1}{\sqrt{x^{2}+y^{2}}}, \quad \frac{1}{E^{*}}=\frac{1-v_{1}^{2}}{E_{1}}+\frac{1-v_{2}^{2}}{E_{2}}
$$

in which $E_{i}, v_{i}, i=1,2$ are the elastic moduli and Poisson ratios of the two contacting surfaces. If the initial gap between these two surfaces before the deformation is denoted by $h(x, y)$, the gap after deformation can be calculated as:

$$
g(x, y)=u(x, y)+h(x, y)-\delta
$$

where $\delta$ is the rigid approach of the two surfaces. All the operations in Eqs. (13-15) are carried out numerically over the discretized domain [16].

All adhesive force components are (directly or indirectly) functions of the gap at the interface and are computed in step 4. According to Eq. (12), the solid-solid van der Waals interaction, given by a constant negative stress $-\sigma_{0}$, is present at areas with gap smaller than $h_{0}$. In all the meniscus wetted areas, the capillary pressure, $\Delta P_{c a p}$ is set and finally, the water-solid van der Waals interaction is present in either of $\Omega_{w s}^{1}$ or $\Omega_{w s}^{2}$.

In step 5, the pressure is updated by balancing the force corresponding the current pressure (including the new adhesive components) with the external normal force. Step 6 checks whether the pressure has converged. If so, the iteration loop stops; otherwise, it starts over from step 3 using the new pressure. The base of this algorithm and the details for updating the pressure is presented in [16]. Here, however, the water-solid interaction and capillary forces in steps 4.2 and 4.3 are embedded in that algorithm. 


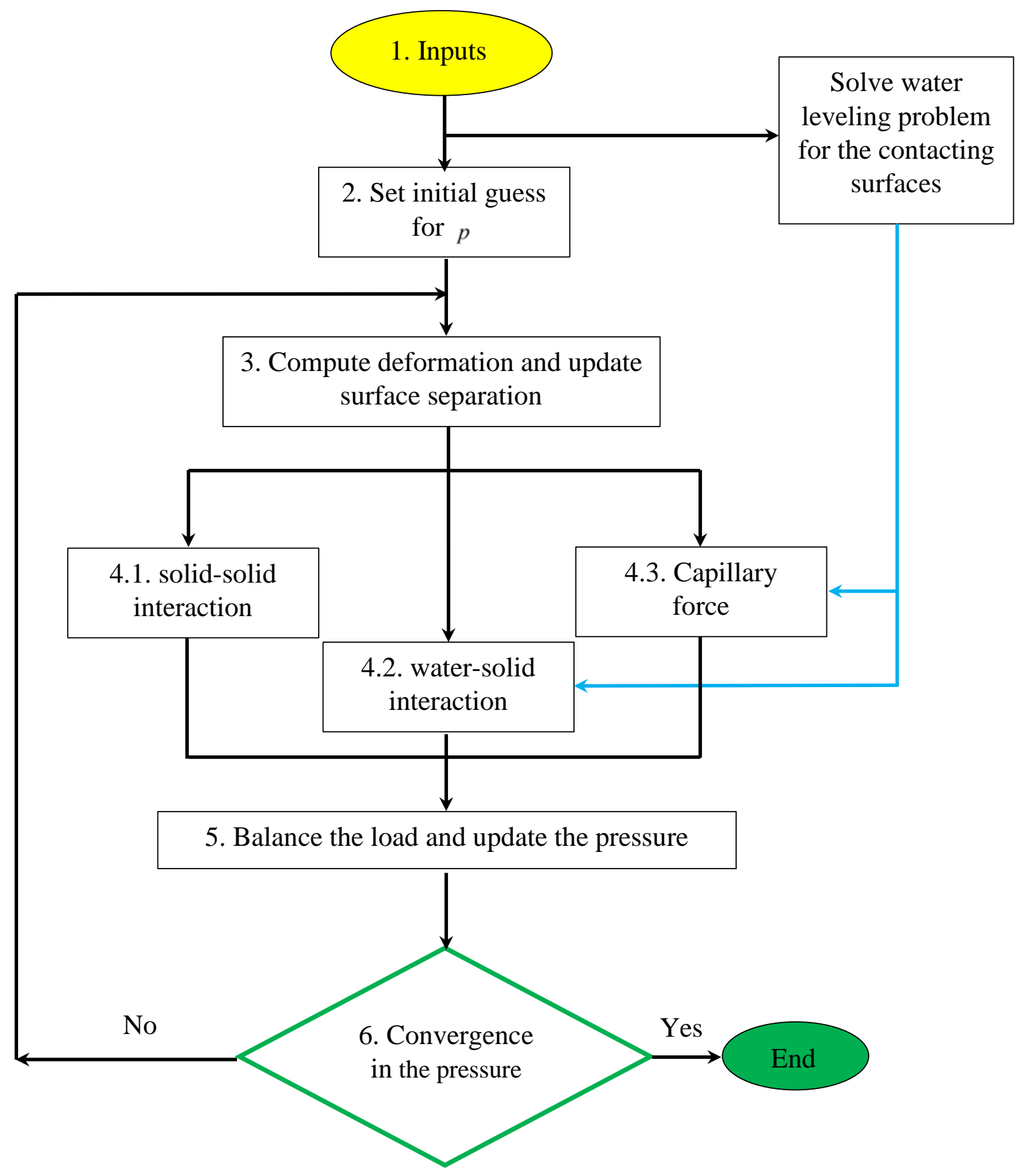

Fig. 6. Numerical scheme to solve the adhesive normal contact problem. 


\section{Numerical examples and discussion}

This section provides two numerical examples. In both examples, the adsorption isotherm of water on the hydrophilic surface of Silicon-Oxide $\left(\mathrm{SiO}_{2}\right)$, measured by Asay and Kim [51], is used to express the average thickness of the water film as a function of $R H$. It is reported that at $R H$ ranging from 0 to $30 \%$, water grows up to three monolayers of hydrogen-bonded ice molecules (Region A in Fig. 7). Then, the liquid structure of water starts to appear and the icelike structure grows to saturation for $R H$ up to $60 \%$ (the transition regime, Region B in Fig. 7). Above $60 \%$ of $R H$, the adsorbed water is considered to be bulk and continues to increase in thickness (Region C in Fig. 7). The water meniscus is expected to form in this region.

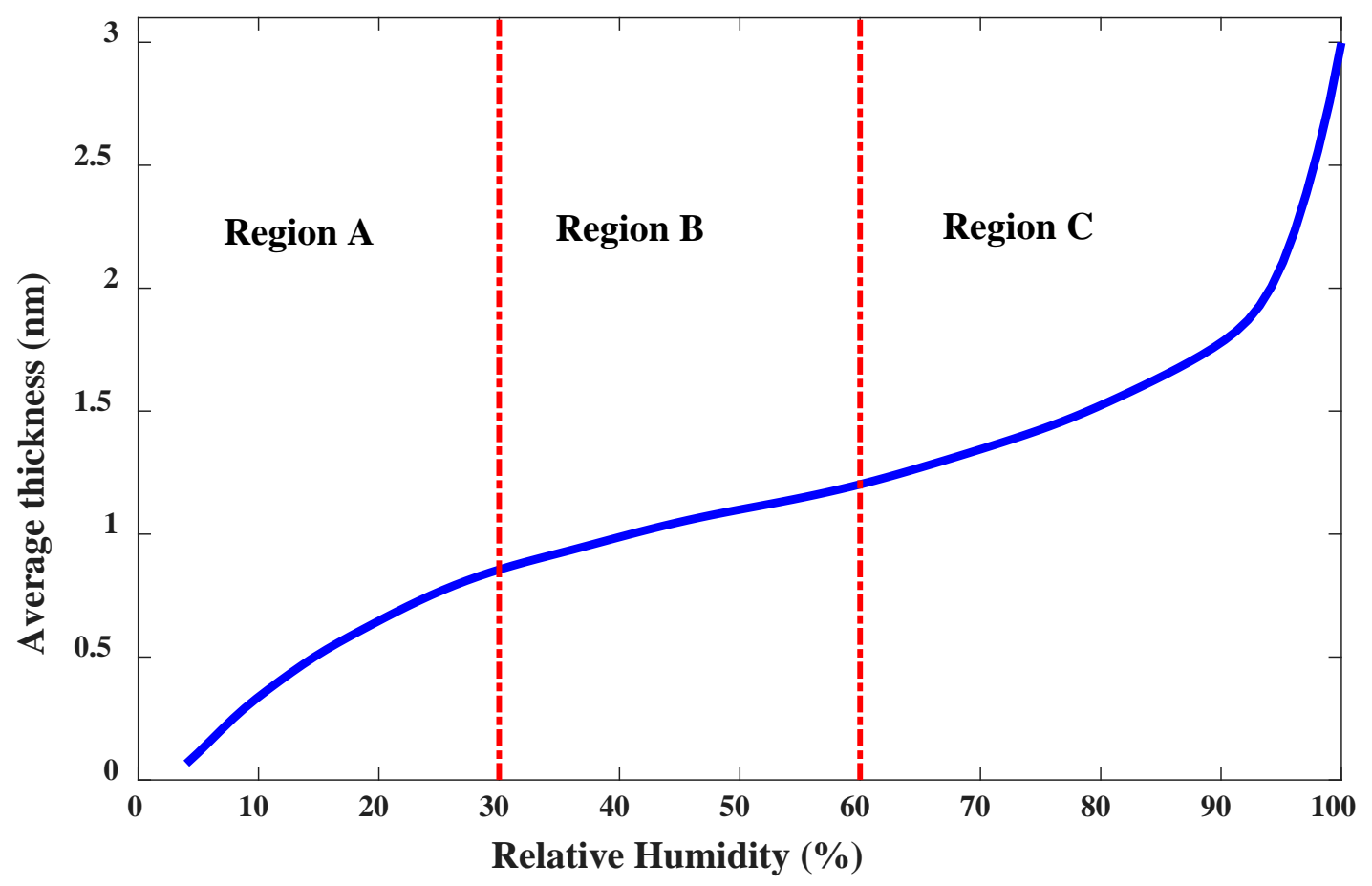

Fig. 7. Adsorption isotherm of water on a silicon-oxide surface (redrawn from Ref. [51]). 
In the following examples, we first examine the accuracy of the proposed BEM model by evaluating the well-known capillary force for the ball-on-flat configuration. Then, the formation of micro-menisci at a rough interface will be investigated.

\subsection{Ball-on-flat configuration}

Fig. 8 displays the formation of a meniscus at the interface of a ball-on-flat configuration in the presence of a thin adsorbed water film. As it was mentioned, the curvature of the meniscus does not significantly change the thickness of the adsorbed film [46].

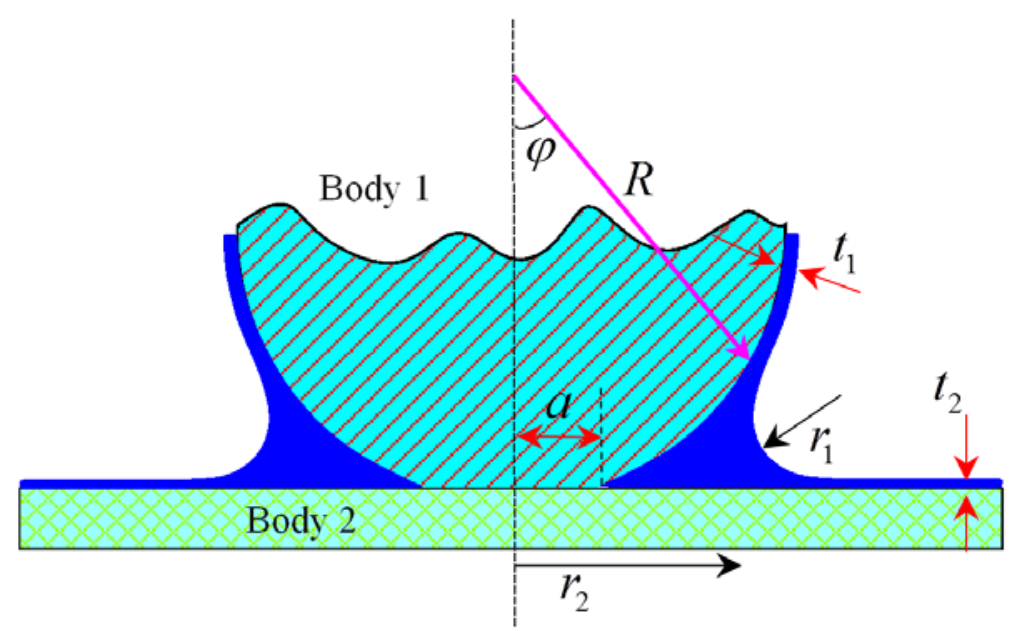

Fig. 8. Meniscus formation at a smooth ball-on-flat interface in the presence of an adsorbed water film.

The meniscus radii of curvature in the presence of a thin adsorbed water film with thickness of $t_{1}$ and $t_{2}$ on the ball and flat, respectively, can be simply obtained as: 


$$
\begin{aligned}
& r_{1}=\frac{R(1-\cos \varphi)-\frac{a^{2}}{R}-\left(t_{1} \cos \varphi+t_{2}\right)}{1+\cos \varphi}, \\
& r_{2}=R \sin \varphi
\end{aligned}
$$

where $a$ and $\varphi$ are the contact radius and the meniscus filling angle, respectively. The term $a^{2} / R$ counts as the normal indentation. Substituting Eq. (16) in Eq. (5) reads:

$$
\frac{1}{R \sin \varphi}-\frac{1+\cos \varphi}{R(1-\cos \varphi)-\frac{a^{2}}{R}-\left(t_{1} \cos \varphi+t_{2}\right)}=\frac{R_{g} T}{\gamma V} \log R H
$$

Solving this equation for the filling angle, $\varphi$, gives the capillary force as:

$$
F_{\text {cap }}=\pi\left(R^{2} \sin ^{2} \varphi-a^{2}\right) \Delta P_{\text {cap }}
$$

It is worth noting that the contact radius, $a$, in Eq. (17) is unknown and dependent on the capillary force (and the filling angle) as well as some other parameters. To the best of our knowledge, however, there is no analytical solution for this problem in the presence of adsorbed films. Here we consider both the ball and flat to be rigid in order to have a zero contact radius and therefore, only does the filling angle remain to be solved and used in Eq. (18). The adsorption isotherms for the ball and flat are considered to be that of $\mathrm{SiO}_{2}$. One must know that the effect of van der Waals forces in Eq. (17) is not considered. Therefore, we also neglect its contribution in the proposed model for this problem.

This problem is solved using the proposed model for two different values of the ball radius. Fig. 9 compares the capillary force obtained through the analytical solution of Eq. (17) and the proposed model. The relative error in both cases and at any $R H$ value is negligible implying that the numerical results are in good agreement with the analytical solution. 

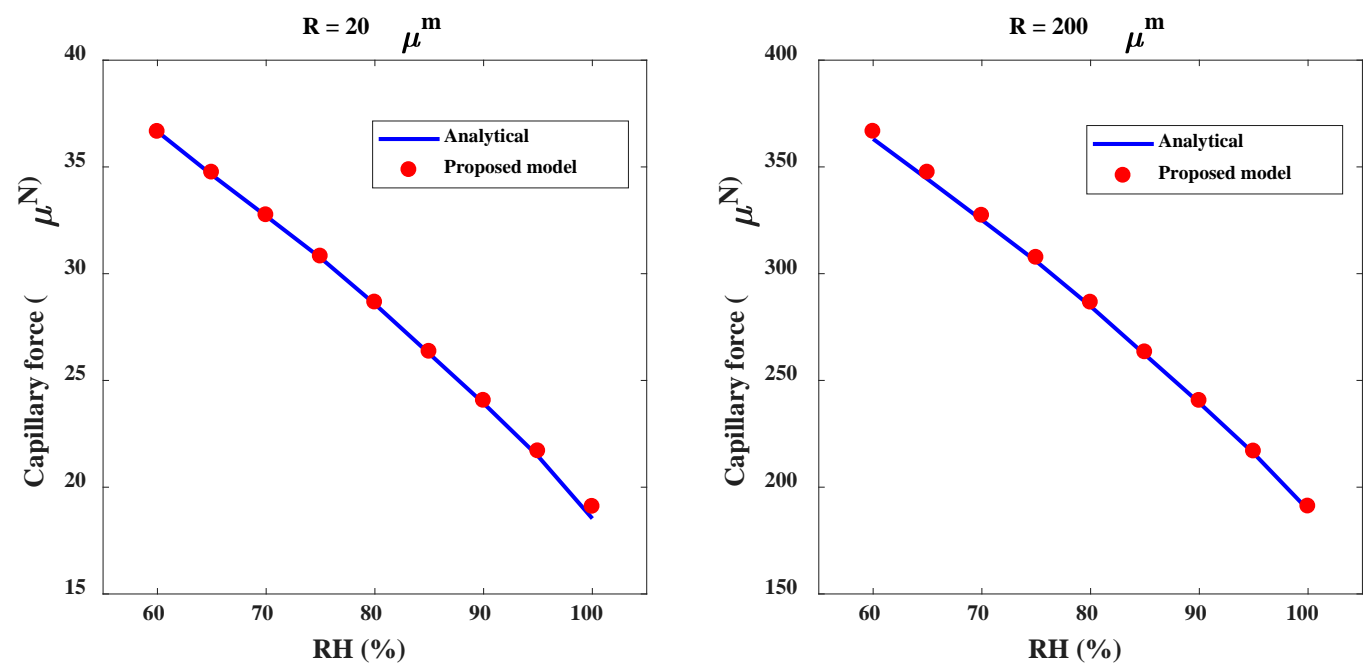

Fig. 9. Comparison of the analytical and numerical solutions for the capillary force at different RH for two different ball radii $(R)$.

Here we conclude that the capillary problem for an elastic ball-on-flat configuration can be numerically solved using the proposed model where the contact radius and filling angle are both automatically derived from the numerical results.

\subsection{Rough interface}

This section aims at studying the adhesive contact at a rough interface. Three Gaussian rough surfaces with different autocorrelation lengths, $\mathrm{L}_{\mathrm{ac}}$, and the same rms value of $\sigma=5 \mathrm{~nm}$, shown in Fig. 10, are generated. The size of each profile is $L \times L=10 \mu \mathrm{m} \times 10 \mu \mathrm{m}$ and it includes $256 \times 256$ nodes which is typical of the surface topography measurement carried out by an Atomic Force Microscopy (the reader is referred to [52,53] for the effect of resolution on the contact area). Each of these surfaces comes into contact with a rigid flat surface. The properties of silicon-oxide $\left(\mathrm{SiO}_{2}\right)$ are used for the elastic rough surface and the water, for the case studied 
here, is assumed to be adsorbed only on the rough surface and thus, the flat surface is dry. For all of the following simulations, the external normal load is $500 \mu \mathrm{N}$ unless otherwise it is mentioned.

(a) $\mathrm{L}_{\text {ac }} / \mathrm{L}=\mathbf{0 . 0 2}$

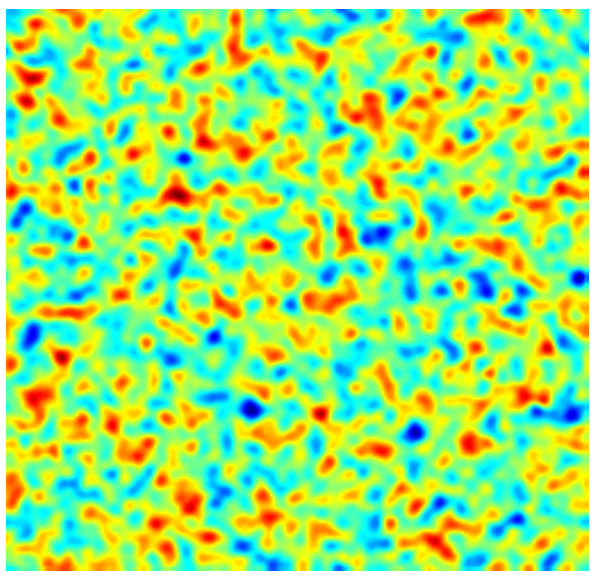

(b) $\mathrm{L}_{\text {ac }} / \mathrm{L}=\mathbf{0 . 0 5}$

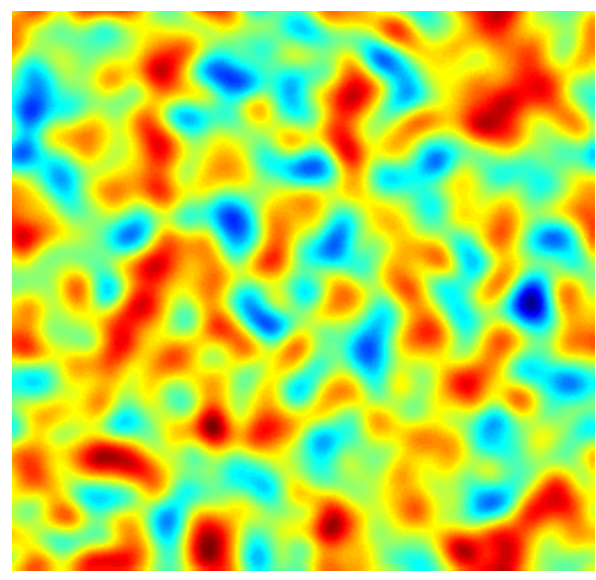

$/ \mathrm{L}=\mathbf{0 . 1 0}$

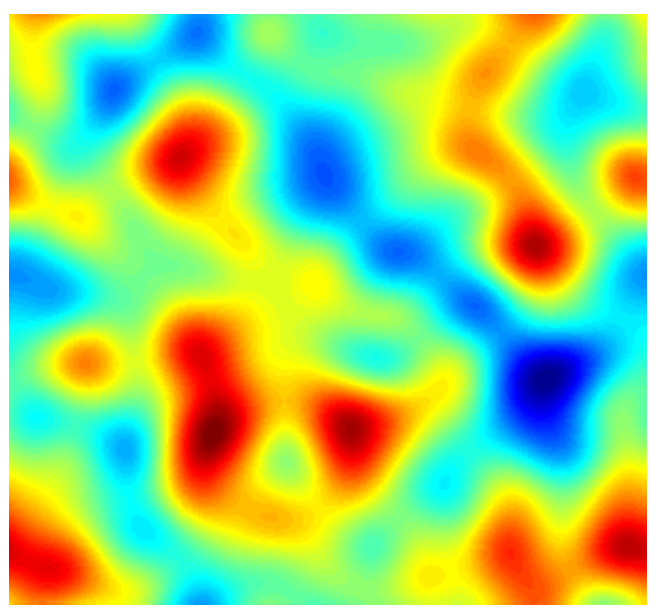

Fig. 10. The roughness height (in $\mathrm{nm}$ ) of the three generated Gaussian isotropic rough surfaces with different autocorrelation length ratio.

Fig. 11 shows a cross section of the leveled water film on top of the mentioned three rough surfaces at different $R H$ levels. The film appears to be thicker at the peaks for surfaces with long 
auto-correlation length. In other words, the lower local gradients of the surface reduces the tendency of the water to flow towards the valleys.
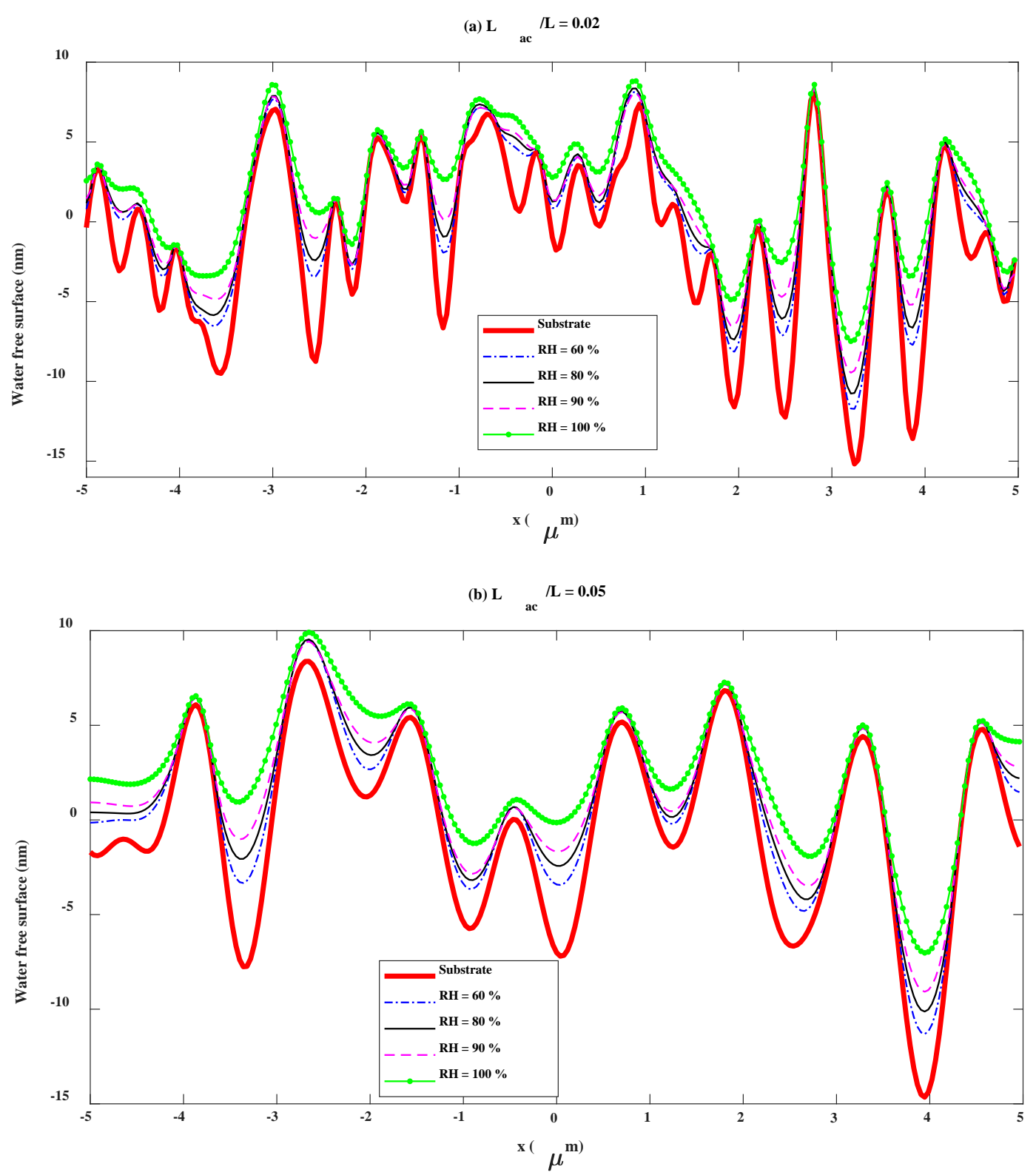


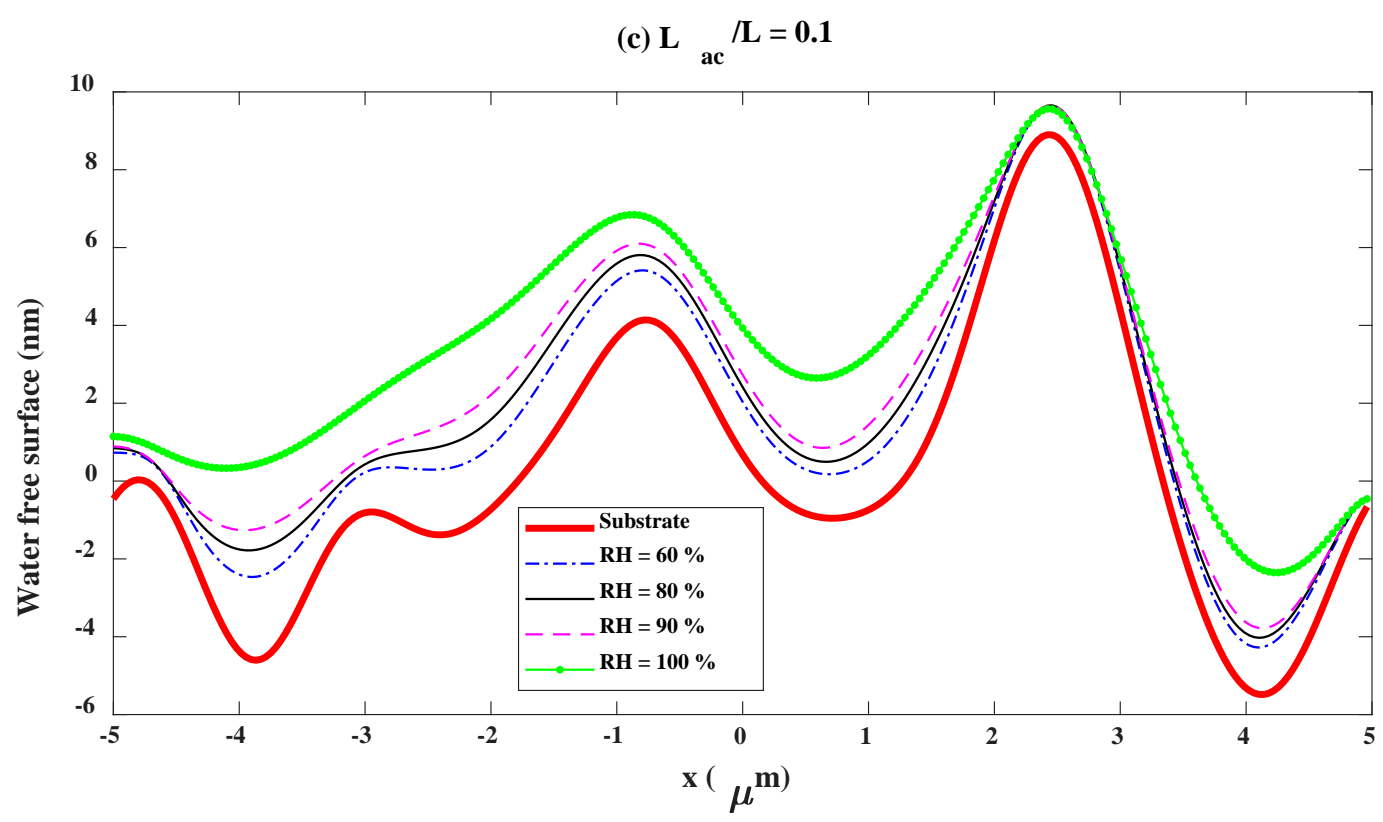

Fig. 11. The leveled water film on the rough surfaces at different RH.

The development of the meniscus area around the contacting regions at the three rough interfaces with increasing the relative humidity is shown in Fig. 12. It is apparent that the meniscus forms right around the contacting and at the near-contacting asperities and develops as the relative humidity increases. The micro-meniscus islands become larger and larger so that they can touch the neighboring micro-menisci and form a larger meniscus. At a high relative humidity level, at around $90 \%$, all these islands are merged to form a single meniscus area which is developed all over the non-contact area. It is worth noting that from $90 \%$ to $100 \%$ of $\mathrm{RH}$, some of the microcontacts are lost. The reason is that, in this range, the capillary force decreases rapidly (it will be further on explained why) and therefore, the contact repulsive force (which is always larger than the external normal force due to the presence of adhesive forces) decreases accordingly and results in a lower contact area. 
$\mathbf{R H}=\mathbf{6 0} \%$

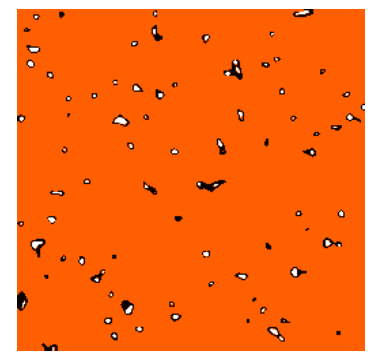




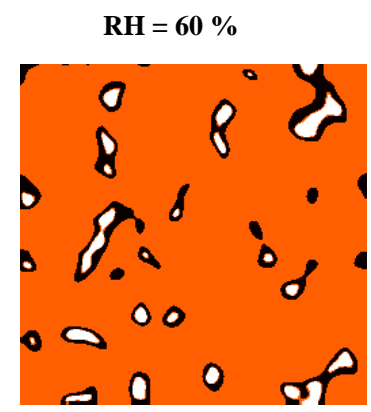




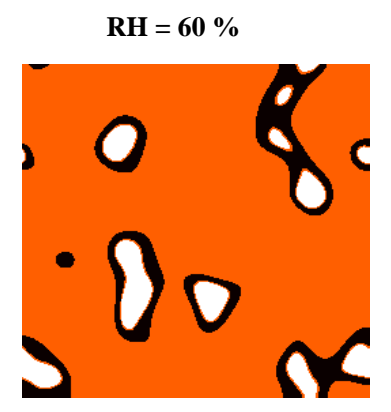

Fig. 12. The development of meniscus area with increasing $\mathrm{RH}$ at the three rough interfaces with different autocorrelation lengths.

The variation of each of the adhesive force components and the total adhesive force at the rough interface with RH is depicted in Fig. 13(a-c). The capillary force first increases to reach its maximum at around $90 \% \mathrm{RH}$ and then rapidly decreases to zero. To explain this behavior, one needs to note that in one hand, the capillary area increases and on the other hand, the capillary pressure, $\Delta P_{\text {cap }}$, decreases with $R H$ and therefore, the capillary force, which is the product of 
these two parameters, is a trade-off between these two changes. Initially, the increase of the capillary area dominates the decrease in the capillary pressure. At higher levels of RH, where most of the non-contact area is covered by the merged micro-menisci, the capillary area does not increase noticeably with $R H$; specifically, after the whole non-contact area is covered with meniscus, there is no room for the capillary area to increase. This is the point where the decrease in the capillary pressure starts to dominate and thus, the capillary force steadily decreases to zero. Looking at Fig. 13(d), which compares the capillary force variation for rough interfaces with different auto-correlation lengths, reveals that the RH at which the maximum capillary force occurs, decreases with the auto-correlation length. In addition, the maximum capillary force increases (however not proportionally) with the auto-correlation length, too. The reason is that the water film is comparatively thicker for surfaces with longer auto-correlation lengths due to the smaller local gradients at the peaks. This figure also implies that at very large $R H$ values, the capillary force is almost the same for all three surfaces and independent from the auto-correlation length due to the fact that all the non-contact area is covered by the meniscus and this parameter is, to a large extent, the same for all these three surfaces.

One should also note that the solid-solid and water-solid interaction forces ( $F_{\text {ss }}$ and $F_{w s}$ respectively) are rather constant with $\mathrm{RH}$, however larger for longer auto-correlation lengths. Furthermore, these two components are quite smaller than the capillary. In other words, the capillary force has the major contribution to the total adhesive force ( $\left.F_{\text {total }}\right)$. 

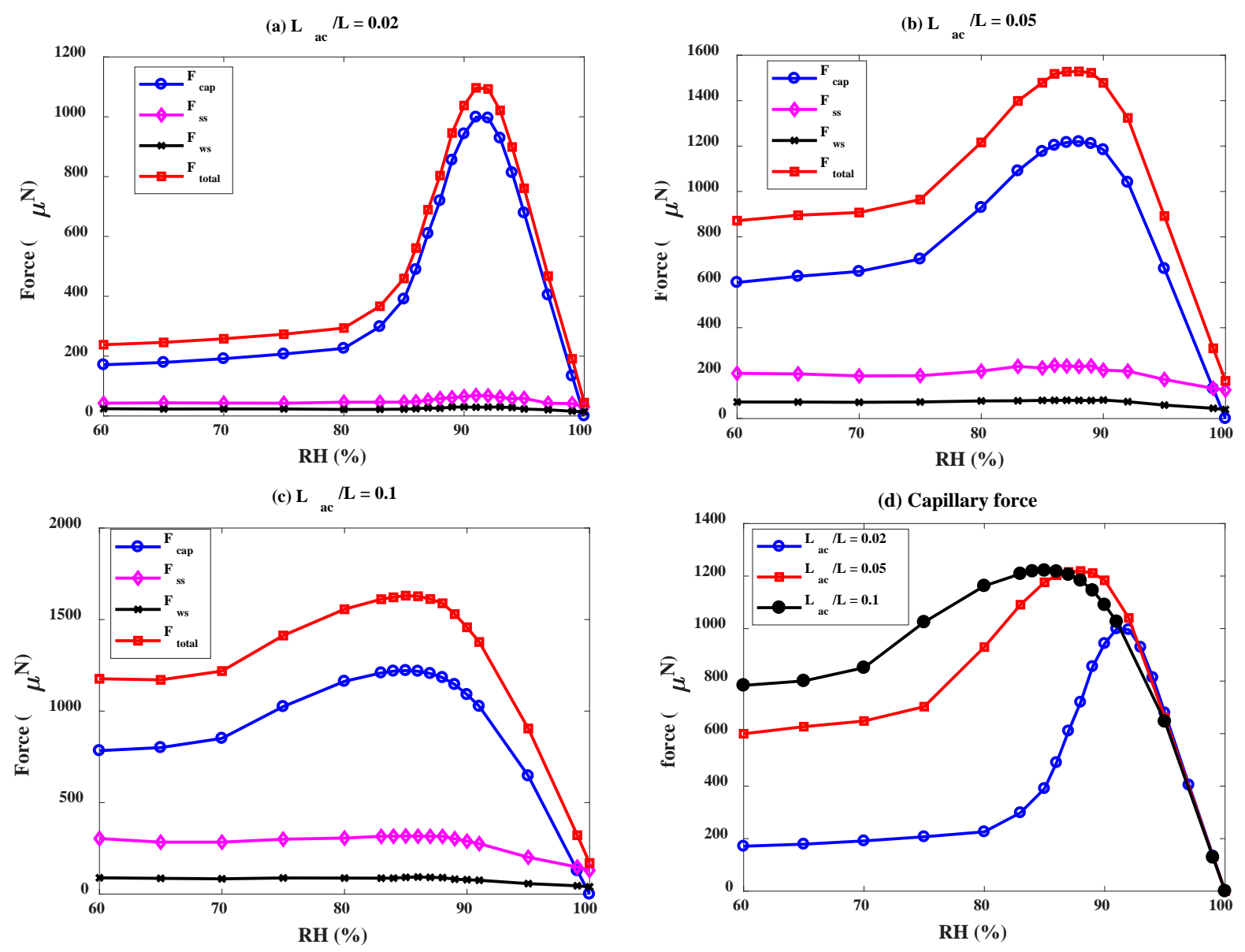

Fig. 13. Variation of different adhesive components vs. RH at three different auto-correlation lengths (a-c) and (d) comparison of the capillary force component.

With the intention to confirm the repeatability of the presented simulations, the capillary development for three different realizations of the same statistical parameters of Fig. 10(a), shown in Fig. 14, is investigated. The variation of the capillary force with RH under $500 \mu \mathrm{N}$ of the normal load is depicted in Fig. 15. As it can be seen, the capillary force for each of the rough interfaces lies within a quite narrow band and they all follow the same curve. Hence, one can conclude that the surface shown in Fig. 10(a) is representative of its corresponding statistical 
parameters and the observed trend for the capillary force holds for other similar surfaces. In addition, similar trends can be expected for surfaces shown in Fig. 10(b and c).

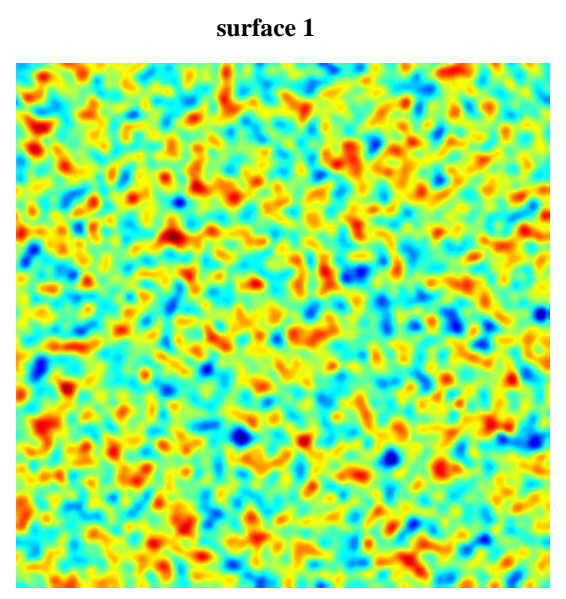

Fig. 14. Roughness heights (in $\mathrm{nm}$ ) of four different Gaussian rough surfaces with identical auto-correlation length ( $\mathrm{L}_{\mathrm{ac}} / \mathrm{L}=0.02$ ), and RMS values $(\sigma=5 \mathrm{~nm})$; surface 1 is the same as Fig. 10(a). 


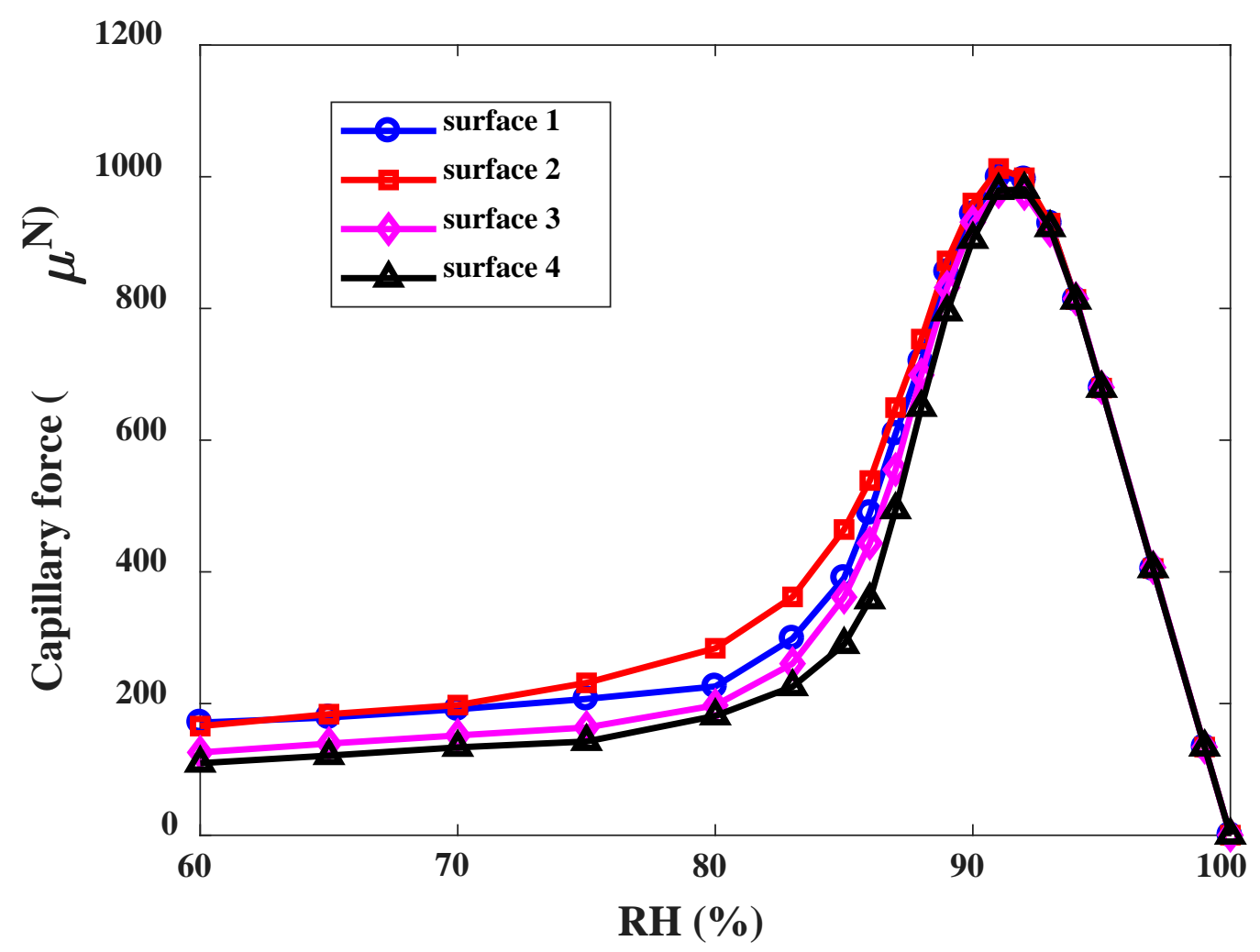

Fig. 15. The comparison of the capillary force for 4 different Gaussian rough surfaces with identical auto-correlation lengths and RMS values ( $\mathrm{L}_{\mathrm{ac}} / \mathrm{L}=0.02, \sigma=5 \mathrm{~nm}$ ).

In order to see how roughness RMS can affect the capillary force variation, the surface shown in Fig. 10(a) is scaled in vertical direction to generate surfaces with RMS values of $7.5 \mathrm{~nm}$ and $10 \mathrm{~nm}$ while the auto-correlation length is kept the same. It is noted that this scaling does not change higher order statistical parameters of the surface such as skewness and kurtosis as long as the arithmetic average of the roughness heights is zero. The variation of the capillary force with $R H$ for these surfaces with different RMS values is illustrated in Fig. 16. This figure suggests that as the RMS values decreases, the capillary force increases and its peak is shifted to a lower $R H$ value. This is, to some extent, similar to the effect of auto-correlation length (see Fig. 13(d)). 
In both cases, smaller local curvatures, due to smaller RMS value and or longer auto-correlation length, results in higher capillary force and shifting the capillary peak to a lower $R H$ value.

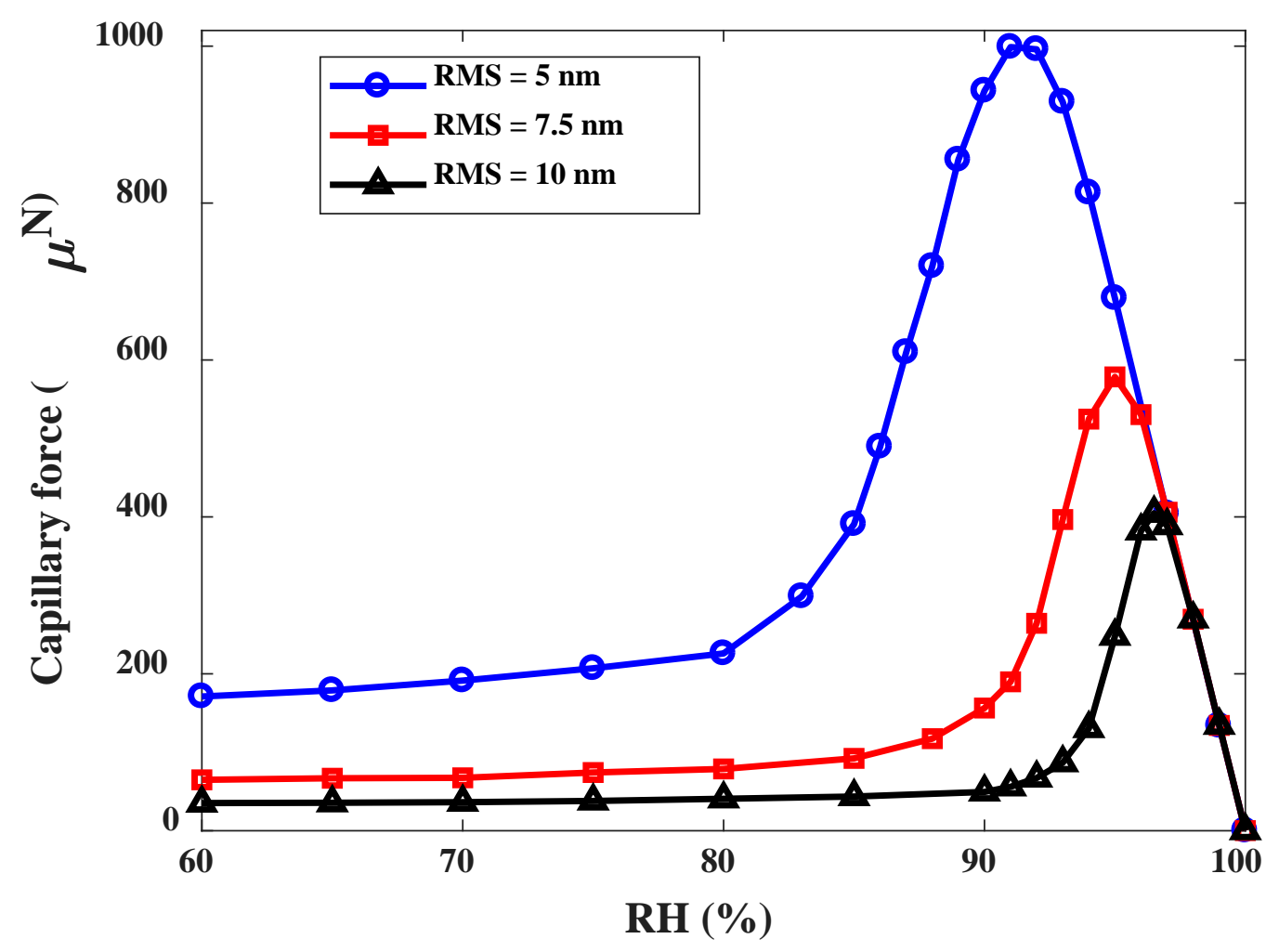

Fig. 16. The comparison of the capillary force for a rough interface with different RMS values.

Another parameter that affects the capillary force at the contact of two rough surfaces is surface deformation. The more the surfaces deform and get closer to one another, the higher chance for larger micro-menisci to form and thus, a larger capillary force. Fig. 17 shows the variation of the capillary force at the three rough interfaces of Fig. 10 for three quite different values of the external normal load. It appears conspicuous that the normal load can change not only the peak location (the corresponding $R H$ value) and value of the capillary force but also the whole shape 
of the curve. In other words, it can change the width of the peak as well; specifically, Fig. 17(c) implies that there is no increase in the capillary force with $R H$ for the normal load of $5000 \mu N$ since the deformation is so large and the contacting surfaces get so close that even at $60 \%$ of $R H$ the major part of the contact is covered by meniscus and any further increase in RH does not remarkably change it so that the decrease in the capillary pressure steadily decreases the capillary force, too. At very high $R H$ values (over 90\%), the capillary force due to different normal loads is slightly different which is due to the minor difference in the non-contact area.
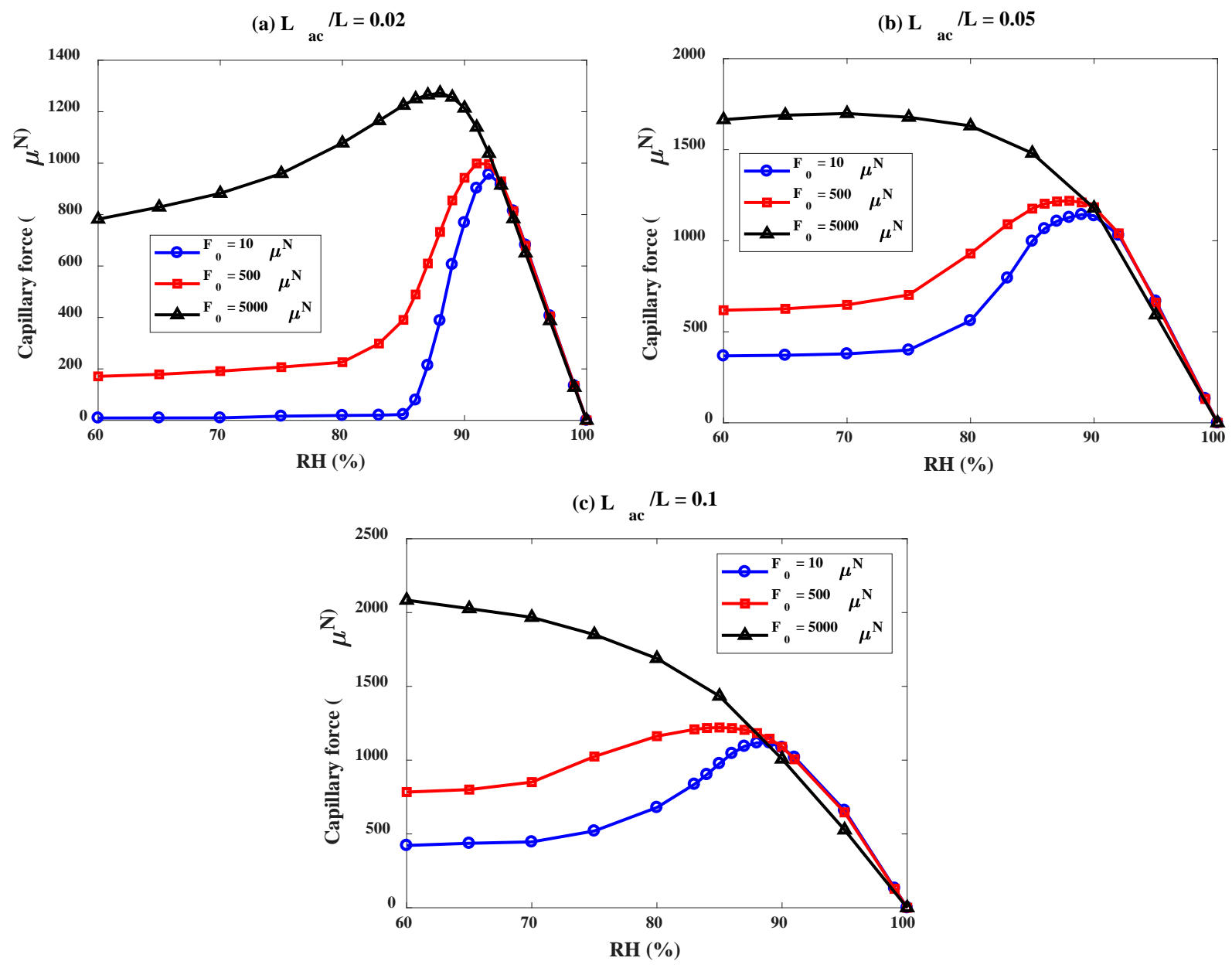

Fig. 17. The effect of external normal load on the capillary force. 
One of the most significant steps in the proposed model is to find the uneven distribution of the water films on the contacting surfaces as described in section 2.2. Here, we are going to investigate how much the capillary force can deviate if we assume a uniform distribution of the water films. When the distribution is uniform, the water film is thicker at the high asperities. This is much more prominent for surfaces with shorter auto-correlation lengths due to the large local gradients which force the water flow towards the valleys. Consequently, at lower $R H$ values, the probability of forming micro-menisci around non-contact asperities is higher. In addition, as the film is thicker around the contacting asperities (compared to the uneven distribution), the micromenisci islands around these asperities develop to a farther distant. Combining these two effects, at lower $R H$ values, a larger capillary force is expected when the water film distribution is uniform. This point can be easily noticed in Fig. 18 which compares the capillary force variation with $R H$ between the variable and uniform thickness for the three rough interfaces of Fig. 13 under $500 \mu \mathrm{N}$ of the normal load. At larger $R H$ values, micro-menisci become larger so that they can merge and form larger unified menisci. At such values, the surface deformations are greater (due to larger capillary force) so that the accumulated water in the valleys can touch the counter surface and help menisci develop to a farther distant. Considering the fact that there is more water accumulated in the valleys when the uneven distribution of the water film is implemented, the resulting capillary area (and therefore the capillary force) is greater. These two regimes are less dominant as the auto-correlation lengths increase since the difference between uneven distribution and the uniform thickness is less (as explained before). For very large $R H$ values, over $90 \%$, there is no significant difference between the calculated capillary forces as almost all of the non-contact area is covered by a meniscus. 

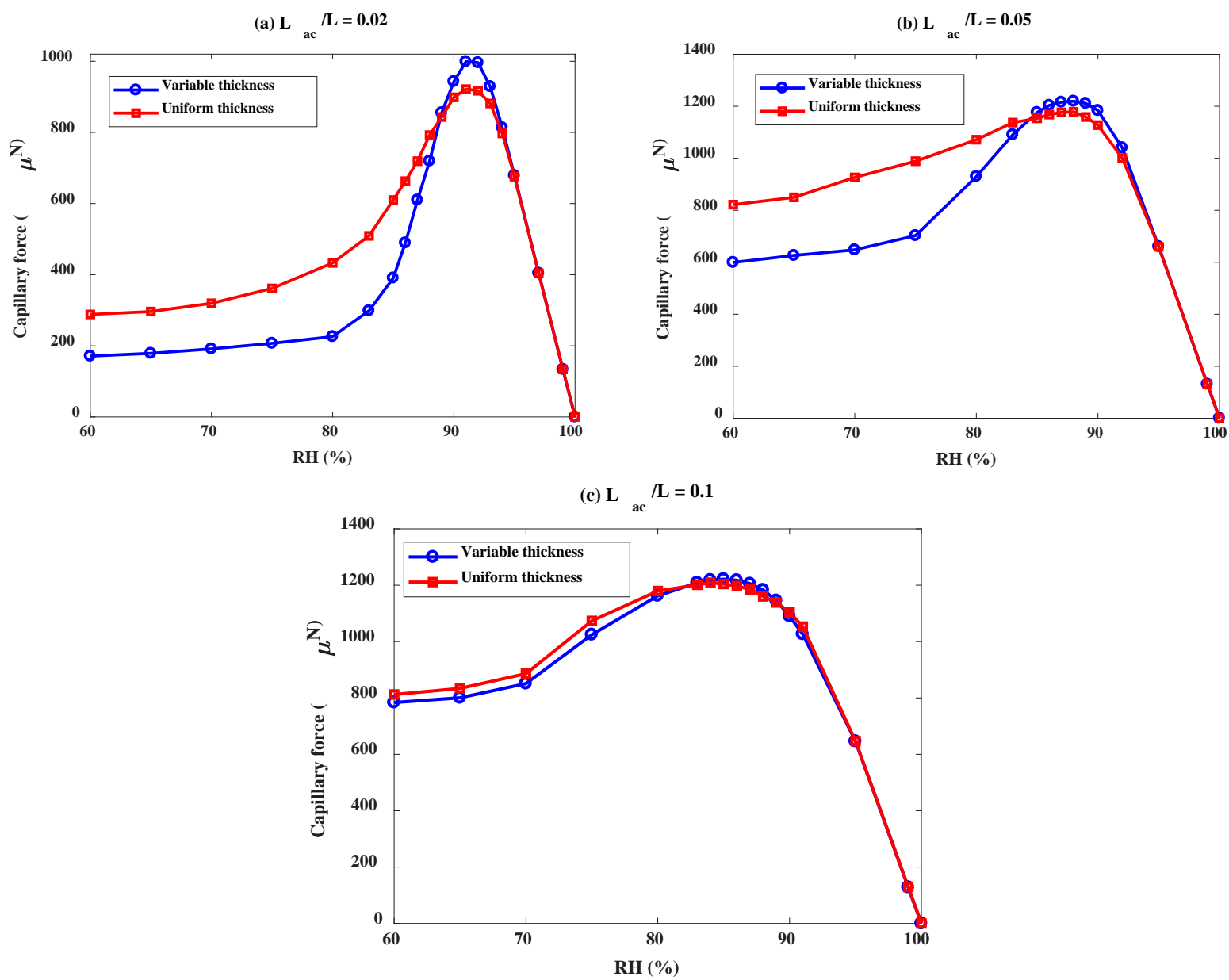

Fig. 18. The comparison of the capillary force with uniform and variable thickness of the water film over the rough surfaces.

\section{Conclusions}

A boundary element model for the adhesive contact of two rough surfaces in the presence of a thin water film adsorbed on the contacting surface is proposed. The distribution of this water film was considered to be uneven over the rough surface. Three different adhesive force components were considered. The capillary force, as the major contributor to the total adhesive force, 
appeared to dominate the solid-solid and water-solid interaction forces. In addition, the capillary force first increased with $R H$ and then decreased as a results of variation in both capillary area and capillary pressure. The maximum of the capillary force was found to be larger and at a lower $R H$ for surfaces with smaller curvatures (here longer auto-correlation length and or smaller roughness rms). The normal force also appeared to be a key factor as it can change not only the maximum capillary force and the corresponding $R H$ but also the entire curve of capillary force versus RH.

\section{Acknowledgment}

The authors would like to express their gratitude to ASML Company, Veldhoven, the Netherlands (www.asml.com) for the financial support of this research project. This research was carried out under project number S61.7.13492 in the framework of the Partnership Program of the Materials innovation institute M2i (www.m2i.nl) and the Netherlands Organization for Scientific Research NWO (www.nwo.nl).

\section{References}

[1] Bradley RS. The cohesive force between solid surfaces and the surface energy of solids. Philos Mag J Sci 1932;86:853-862.

[2] Johnson KL, Kendall K, Roberts AD. Surface Energy and the Contact of Elastic Solids. Proc R Soc Lond A Math Phys Sci 1971;324:301-313.

[3] Derjaguin B V, Muller VM, Toporov YP. Effect of contact deformations on the adhesion of particles. J Colloid Interface Sci 1975;53:314-26.

[4] Maugis D. Adhesion of spheres: The JKR-DMT transition using a dugdale model. J 
Colloid Interface Sci 1992;150:243-69. doi:10.1016/0021-9797(92)90285-T.

[5] Song Z, Komvopoulos K. Adhesive contact of an elastic semi-infinite solid with a rigid rough surface: Strength of adhesion and contact instabilities. Int J Solids Struct 2014;51:1197-207. doi:10.1016/j.ijsolstr.2013.10.039.

[6] Götzinger M, Peukert W. Particle adhesion force distributions on rough surfaces. Langmuir 2004;20:5298-303. doi:10.1021/la049914f.

[7] Cho SS, Park S. Finite element modeling of adhesive contact using molecular potential. Tribol Int 2004;37:763-9. doi:10.1016/j.triboint.2004.04.007.

[8] Sauer RA. A Computational Model for Nanoscale Adhesion between Deformable Solids and Its Application to Gecko Adhesion. J Adhes Sci Technol 2010;24:1807-18. doi:10.1163/016942410X507588.

[9] Carbone G, Pierro E. The Influence of the Fractal Dimension of Rough Surfaces on the Adhesion of Elastic Materials. J Adhes Sci Technol 2012;26:2555-70. doi:10.1163/156856111X623140.

[10] Ciavarella M. A note on the possibility of roughness enhancement of adhesion in Persson’s theory. Int J Mech Sci 2017;121:119-22.

[11] Greenwood JA, Williamson JPB. Contact of Nominally Flat Surfaces. Proc R Soc London Ser A , Math Phys Publ 1966;295:300-19.

[12] Muser MH. A dimensionless measure for adhesion and effects of the range of adhesion in contacts of nominally flat surfaces. Tribol Int 2015:1-7.

doi:10.1016/j.triboint.2015.11.010.

[13] Mulakaluri N, Persson BNJ. Adhesion between elastic solids with randomly rough surfaces: Comparison of analytical theory with molecular-dynamics simulations. EPL (Europhysics Lett 2011;96:66003. doi:10.1209/0295-5075/96/66003.

[14] Yang C, Persson BNJ, Israelachvili JN, Rosenberg K. Contact mechanics with adhesion: Interfacial separation and contact area. Europhys Lett 2008;84:5. doi:10.1209/02955075/84/46004.

[15] Rey V, Anciaux G, Molinari J-F. Normal adhesive contact on rough surfaces: efficient algorithm for FFT-based BEM resolution. Comput Mech 2017;60:69-81. doi:10.1007/s00466-017-1392-5.

[16] Bazrafshan M, de Rooij MB, Valefi M, Schipper DJ. Numerical method for the adhesive normal contact analysis based on a Dugdale approximation. Tribol Int 2017;112:117-28. doi:10.1016/j.triboint.2017.04.001.

[17] Rimai DS, Quesnel DJ, DeMejo LP, Regan MT. Toner to photoconductor adhesion. J Imaging Sci Technol 2001;45:179-86.

[18] Persson BNJ. Capillary adhesion between elastic solids with randomly rough surfaces. J Phys Condens Matter 2008;20:315007. doi:10.1088/0953-8984/20/31/315007.

[19] Ardito R, Frangi A, Rizzini F, Corigliano A. Evaluation of adhesion in microsystems 
using equivalent rough surfaces modeled with spherical caps. Eur J Mech A/Solids 2016;57:121-31. doi:10.1016/j.euromechsol.2015.12.004.

[20] Zarate N, Harrison A, Litster J, Beaudoin S. Effect of relative humidity on onset of capillary forces for rough surfaces. J Colloid Interface Sci 2013;411:265-72.

[21] Prodanov N, Dapp WB, Müser MH. On the Contact Area and Mean Gap of Rough, Elastic Contacts: Dimensional Analysis, Numerical Corrections, and Reference Data. Tribol Lett 2014;53:433-48. doi:10.1007/s11249-013-0282-z.

[22] Ciavarella M. A very simple estimate of adhesion of hard solids with rough surfaces based on a bearing area model. Meccanica 2017:1-10. doi:10.1007/s11012-017-0701-6.

[23] Vakis AI, Yastrebov VA, J. Scheibert, C. Minfray, Nicola L, Dini D, et al. Modeling and simulation in tribology across scales: An overview. Tribol Int 2018. doi:https://doi.org/10.1016/j.triboint.2018.02.005.

[24] Hanies WB. Studies in the physical properties of soils: III. Observations on the electrical conductivity of soils. J Agric Sci 1925;15:536-43.

[25] Fisher RA. On the capillary forces in an ideal soil; correction of formulae given by WB Haines. J Agric Sci 1926;16:492-505. doi:10.1017/S0021859600007838.

[26] De Souza EJ, Brinkmann M, Mohrdieck C, Arzt E. Enhancement of capillary forces by multiple liquid bridges. Langmuir 2008;24:8813-20. doi:10.1021/la8005376.

[27] Gao C. Theory of menisci and its applications. Appl Phys Lett 1997;13:1801. doi:10.1063/1.119403.

[28] Lazzer A De, Dreyer M, Rath HJ. Particle - Surface Capillary Forces 1999:4551-9.

[29] Sirghi L, Nakagiri N, Sugisaki K. Effect of Sample Topography on Adhesive Force in Atomic Force Spectroscopy Measurements in Air. Langmuir 2000:0-4.

[30] Tselishchev YG, Val'tsifer VA. Influence of the type of contact between particles joined by a liquid bridge on the capillary cohesive forces. Colloid J Russ Acad Sci Kolloidn Zhurnal 2003;65:385-9. doi:10.1023/A:1024275327145.

[31] Willett CD, Adams MJ, Johnson SA, Seville JPK. Capillary bridges between two spherical bodies. Langmuir 2000;16:9396-405. doi:10.1021/la000657y.

[32] Gao C, Dai P, Homola A, Weiss J. Meniscus Forces and Profiles: Theory and Its Applications to Liquid-Mediated Interfaces. J Tribol 1998;120:358-68. doi:10.1115/1.2834435.

[33] Xiao X, Qian L. Investigation of humidity-dependent capillary force. Langmuir 2000;16:8153-8. doi:10.1021/la000770o.

[34] Yaqoob MA, de Rooij MB, Schipper DJ. On the transition from bulk to ordered form of water: A theoretical model to calculate adhesion force due to capillary and van der Waals interaction. Tribol Lett 2013;49:491-9. doi:10.1007/s11249-012-0090-х.

[35] Asay DB, Kim SH. Effects of adsorbed water layer structure on adhesion force of silicon 
oxide nanoasperity contact in humid ambient. J Chem Phys 2006;124.

doi:10.1063/1.2192510.

[36] Xue X, Polycarpou AA. An improved meniscus surface model for contacting rough surfaces. J Colloid Interface Sci 2007;311:203-11. doi:10.1016/j.jcis.2007.02.038.

[37] Boer MP. Capillary adhesion between elastically hard rough surfaces. Exp Mech 2007;47:171-83. doi:10.1007/s11340-006-0631-z.

[38] Wang L, Régnier S. A More General Capillary Adhesion Model Including Shape Index: Single-Asperity and Multi-Asperity Cases. Tribol Trans 2014;58:106-12. doi:10.1080/10402004.2014.951751.

[39] You S, Wan MP. Mathematical models for the van der waals force and capillary force between a rough particle and surface. Langmuir 2013;29:9104-17.

doi:10.1021/la401516m.

[40] Peng YF, Guo YB, Hong YQ. An Adhesion Model for Elastic-Contacting Fractal Surfaces in the Presence of Meniscus. J Tribol 2009;131:24504. doi:10.1115/1.3084213.

[41] Tian X, Bhushan B. The micro-meniscus effect of a thin liquid film on the static friction of rough surface contact. J Phys D Appl Phys 1996;29:163-78. doi:10.1088/00223727/29/1/026.

[42] Lin JF, Chen SC. Calculations of the Meniscus Force and the Contact Force Formed in the Microcontacts of a Rough Surface and a Smooth, Rigid Surface with a Thin Water Film. J Mech 2008;24:1-11. doi:10.1017/S1727719100001519.

[43] Rostami A, Streator JL. A deterministic approach to studying liquid-mediated adhesion between rough surfaces. Tribol Lett 2015;58:1-13. doi:10.1007/s11249-015-0497-2.

[44] J.N. Israelachvili. Intermolecular and surface forces. vol. 53. 2013. doi:10.1017/CBO9781107415324.004.

[45] Figliuzzi B, Jeulin D, Lemaître A, Fricout G, Piezanowski J-J, Manneville P. Numerical simulation of thin paint film flow. J Math Ind 2012;2:1. doi:10.1186/2190-5983-2-1.

[46] DelRio FW, Dunn ML, de Boer MP. Capillary adhesion model for contacting micromachined surfaces. Scr Mater 2008;59:916-20. doi:10.1016/j.scriptamat.2008.02.037.

[47] Mecke KR. Thermal fluctuations of thin liquid films. J Phys Condens Matter 2001;13:4615-36. doi:10.1088/0953-8984/13/21/302.

[48] Wang QJ, Chung YW. Encyclopedia of tribology. 2013. doi:10.1007/978-0-387-92897-5.

[49] Butt H-J, Graf K, Kappl M. Physics and chemistry of interfaces. Weinheim: WILEY_VCH GmbH \& Co. KGaA; 2003.

[50] Johnson KL. Contact Mechanics. Cambridge: Cambridge University Press; 1985.

[51] Asay DB, Kim SH. Evolution of the adsorbed water layer structure on silicon oxide at room temperature. J Phys Chem B 2005;109:16760-3. doi:10.1021/jp053042o. 
[52] Ciavarella M, Papangelo A. Discussion of "Measuring and Understanding Contact Area at the Nanoscale: A Review” (Jacobs, T. D. B., and Ashlie Martini, A., 2017, ASME Appl. Mech. Rev., 69 (6), p. 061101). Appl Mech Rev 2017;69:65502. doi:10.1115/1.4038188.

[53] Jacobs T, Martini A. Review : Measuring and Understanding Contact Area at the Nanoscale. Appl Mech Rev 2017;69. doi:10.1115/1.4038130. 


\section{List of Captions:}

Fig. 1. Dugdale approximation of Lennard-Jones potential. (For interpretation of the references to color in this figure legend, the reader is referred to the web version of this article).

Fig. 2. Meniscus formation at a smooth ball-on-flat interface.

Fig. 3. Leveling of a water film over a rough surface.

Fig. 4. (a) Meniscus formation at the contact of two rough surfaces in the presence of adsorbed water films (b) strategy to find meniscus-wetted asperities (c) a schematic diagram of contact area, meniscus-wetted area, and cross-cut area at mean meniscus height.

Fig. 5. Two different scenarios for the water-solid interaction.

Fig. 6. Numerical scheme to solve the adhesive normal contact problem.

Fig. 7. Adsorption isotherm of water on a silicon-oxide surface (redrawn from Ref. [42]).

Fig. 8. Meniscus formation at a smooth ball-on-flat interface in the presence of an adsorbed water film.

Fig. 9. Comparison of the analytical and numerical solutions for the capillary force at different RH for two different ball radii $(\mathrm{R})$.

Fig. 10. The roughness height (in $\mathrm{nm}$ ) of the three generated Gaussian isotropic rough surfaces with different auto-correlation length ratio.

Fig. 11. The leveled water film on the rough surfaces at different RH.

Fig. 12. The development of meniscus area with increasing $R H$ at the three rough interfaces with different auto-correlation lengths.

Fig. 13. Variation of different adhesive components vs. RH at three different auto-correlation lengths (a-c) and (d) comparison of the capillary force component. 
Fig. 14. Four different Gaussian rough surfaces with identical auto-correlation length $\left(L_{a c} / L=0.02\right)$, and RMS values ( $\sigma=5 \mathrm{~nm}$ ); surface 1 is the same as Fig. 10(a).

Fig. 15. The comparison of the capillary force for $\mathbf{4}$ different Gaussian rough surfaces with identical autocorrelation lengths and $\mathbf{R M S}$ values $\left(\mathrm{L}_{\mathrm{ac}} / \mathrm{L}=0.02, \sigma=5 \mathrm{~nm}\right)$.

Fig. 16. The comparison of the capillary force for rough interface with different RMS values.

Fig. 17. The effect of external normal load on the capillary force.

Fig. 18. The comparison of the capillary force with uniform and variable thickness of the water film over the rough surfaces. 


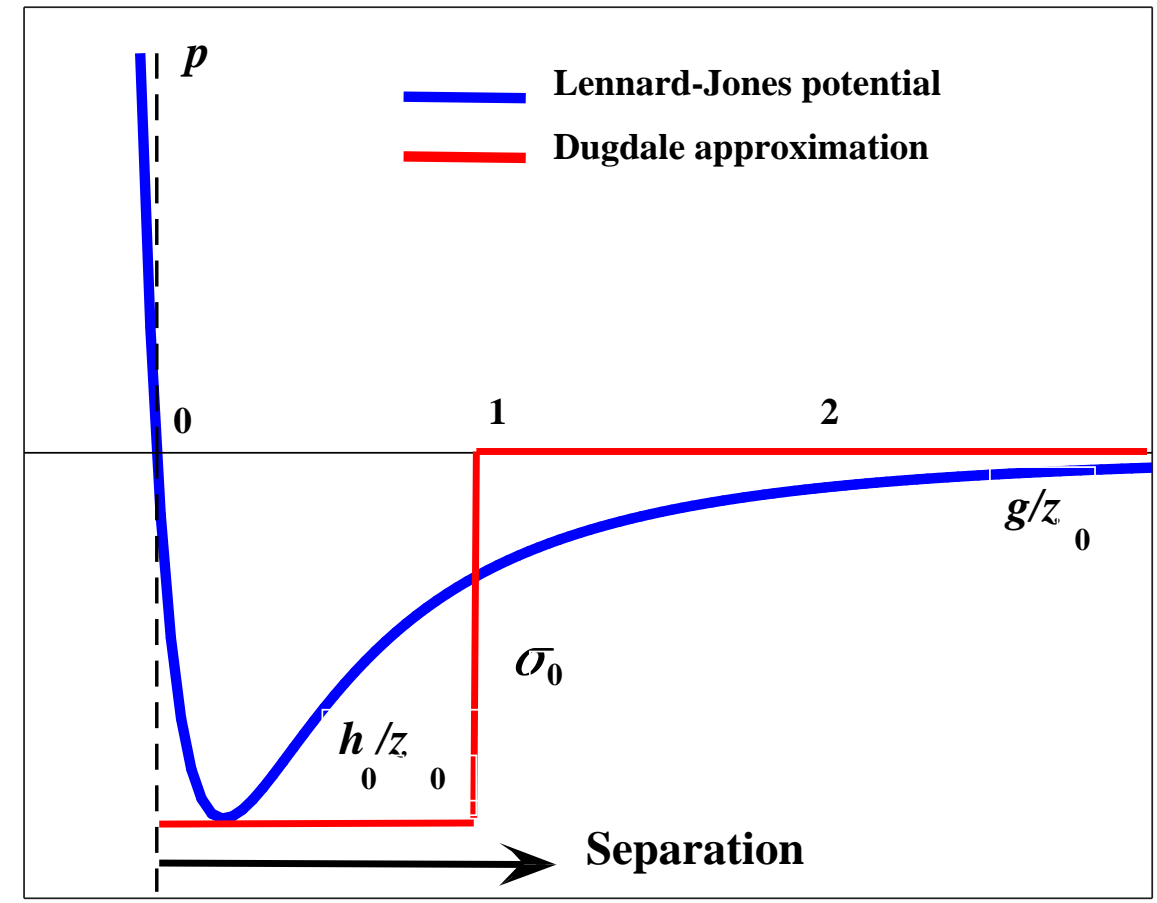

Fig. 1. Dugdale approximation of Lennard-Jones potential. (For interpretation of the references to color in this figure legend, the reader is referred to the web version of this article). 


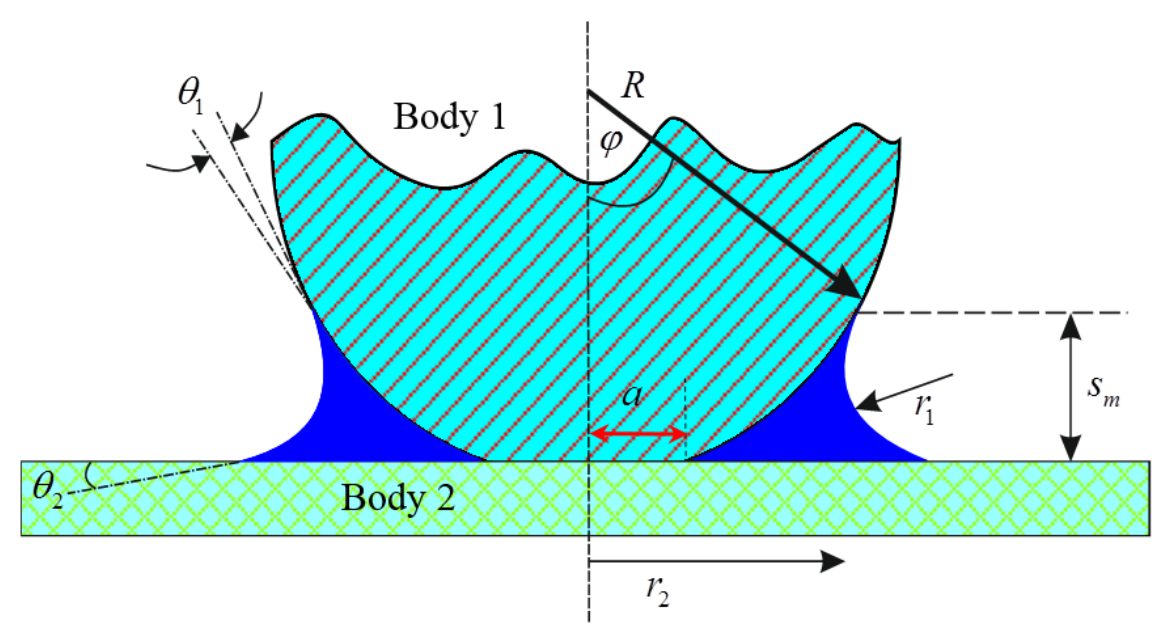

Fig. 2. Meniscus formation at a smooth ball-on-flat interface. 


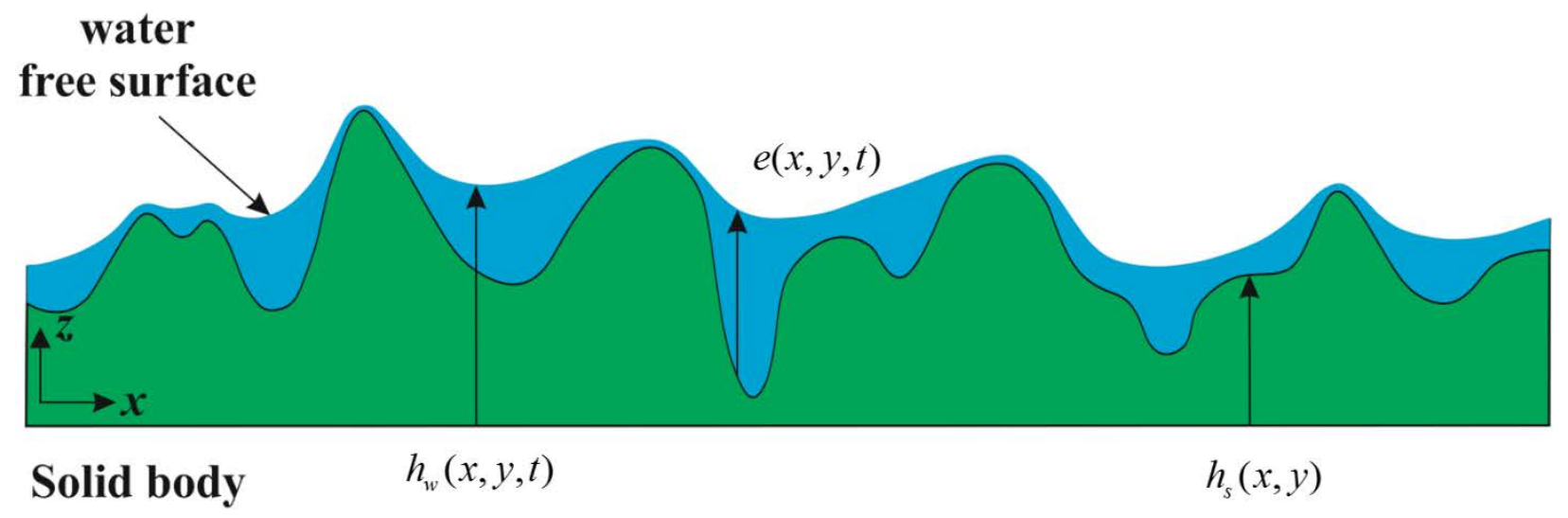

Fig. 3. Leveling of a water film over a rough surface. 
(a)

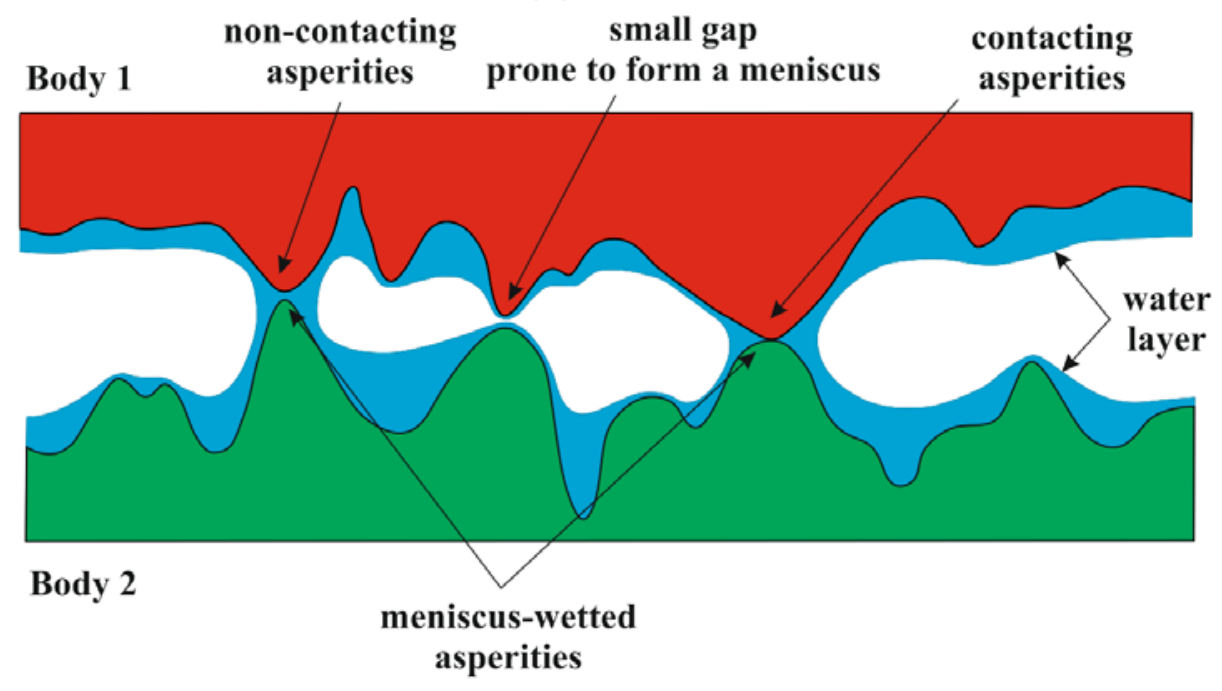

(b)

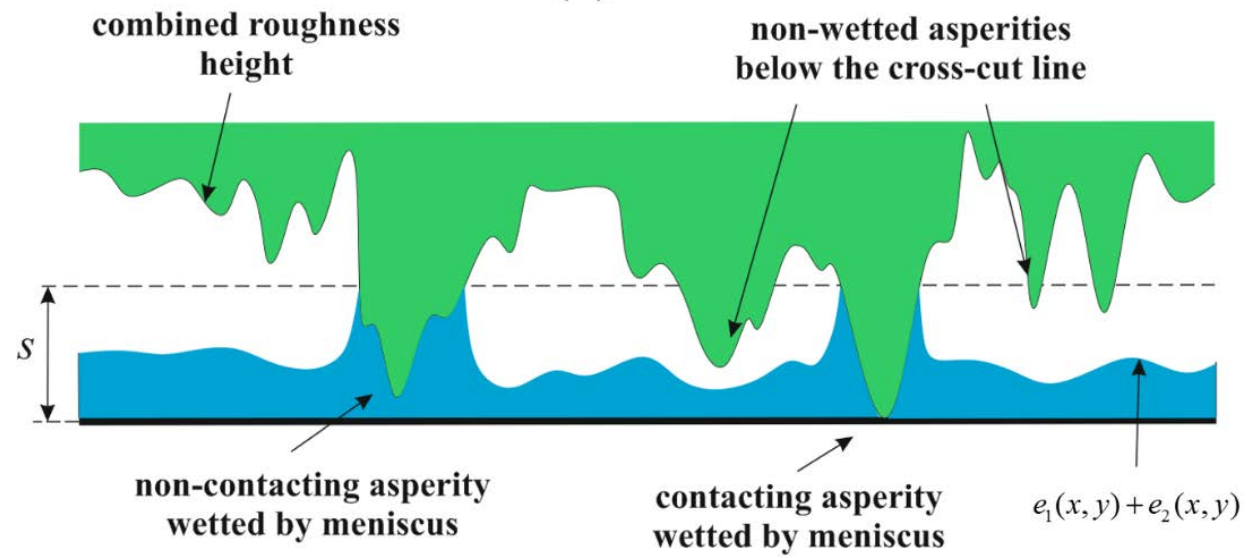

(c) Contact area

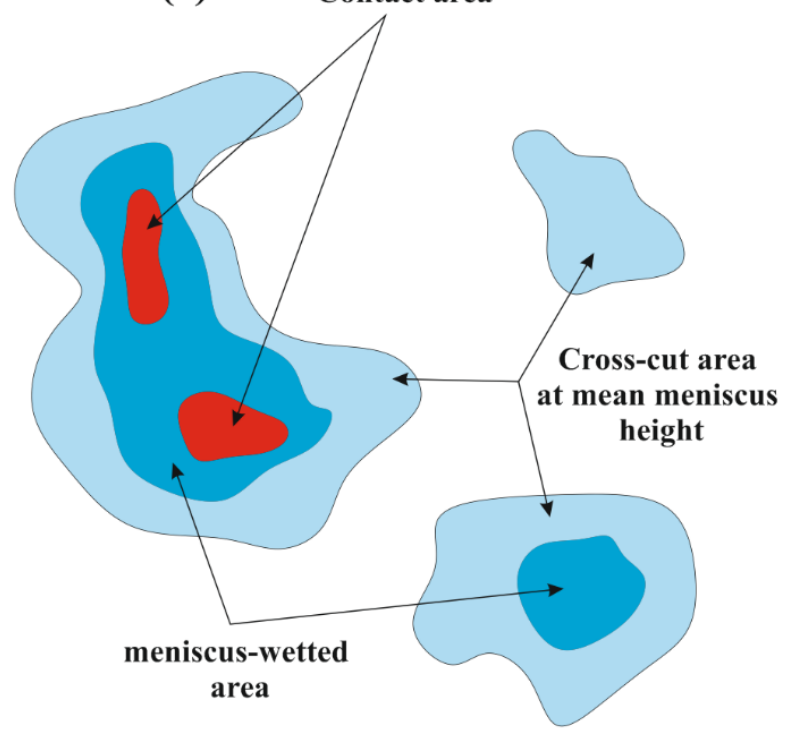


Fig. 4. (a) Meniscus formation at the contact of two rough surfaces in the presence of adsorbed water films (b) strategy to find meniscus-wetted asperities (c) a schematic diagram of contact area, meniscus-wetted area, and crosscut area at mean meniscus height. 
(a)

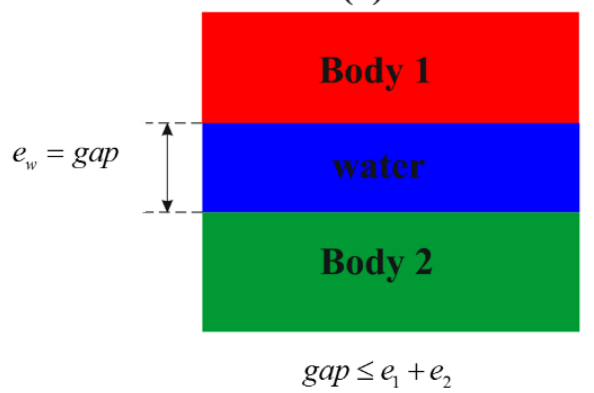

(b)

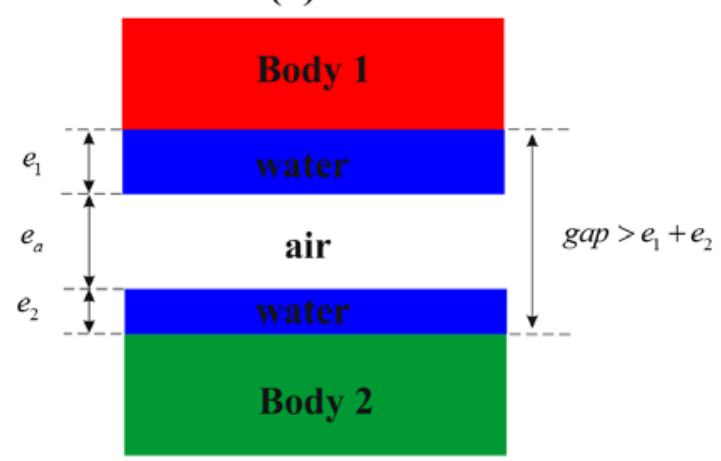

Fig. 6. Numerical scheme to solve the adhesive normal contact problem.Fig. 5. Two different scenarios for the water-solid interaction. 


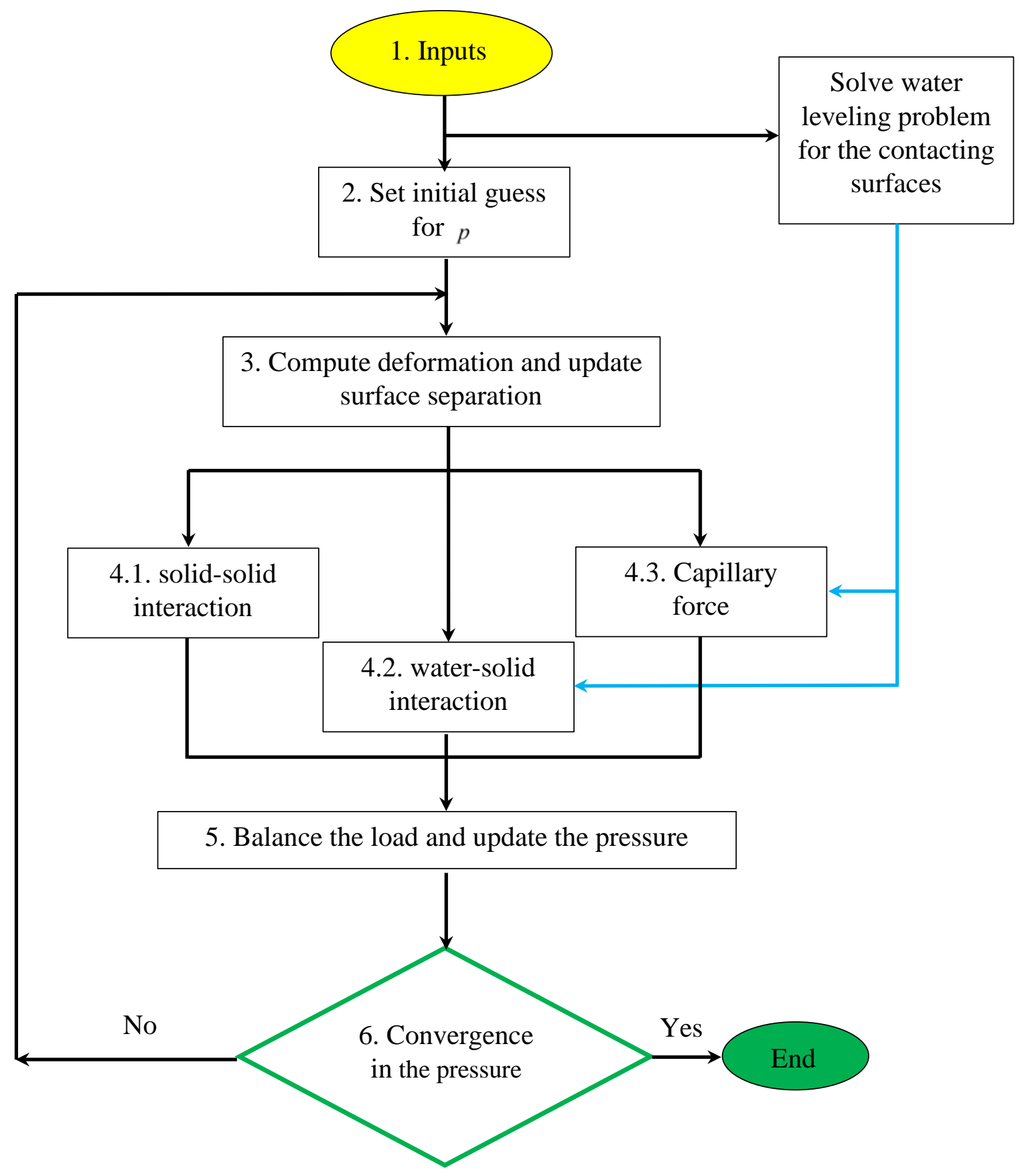

Fig. 6. Numerical scheme to solve the adhesive normal contact problem. 


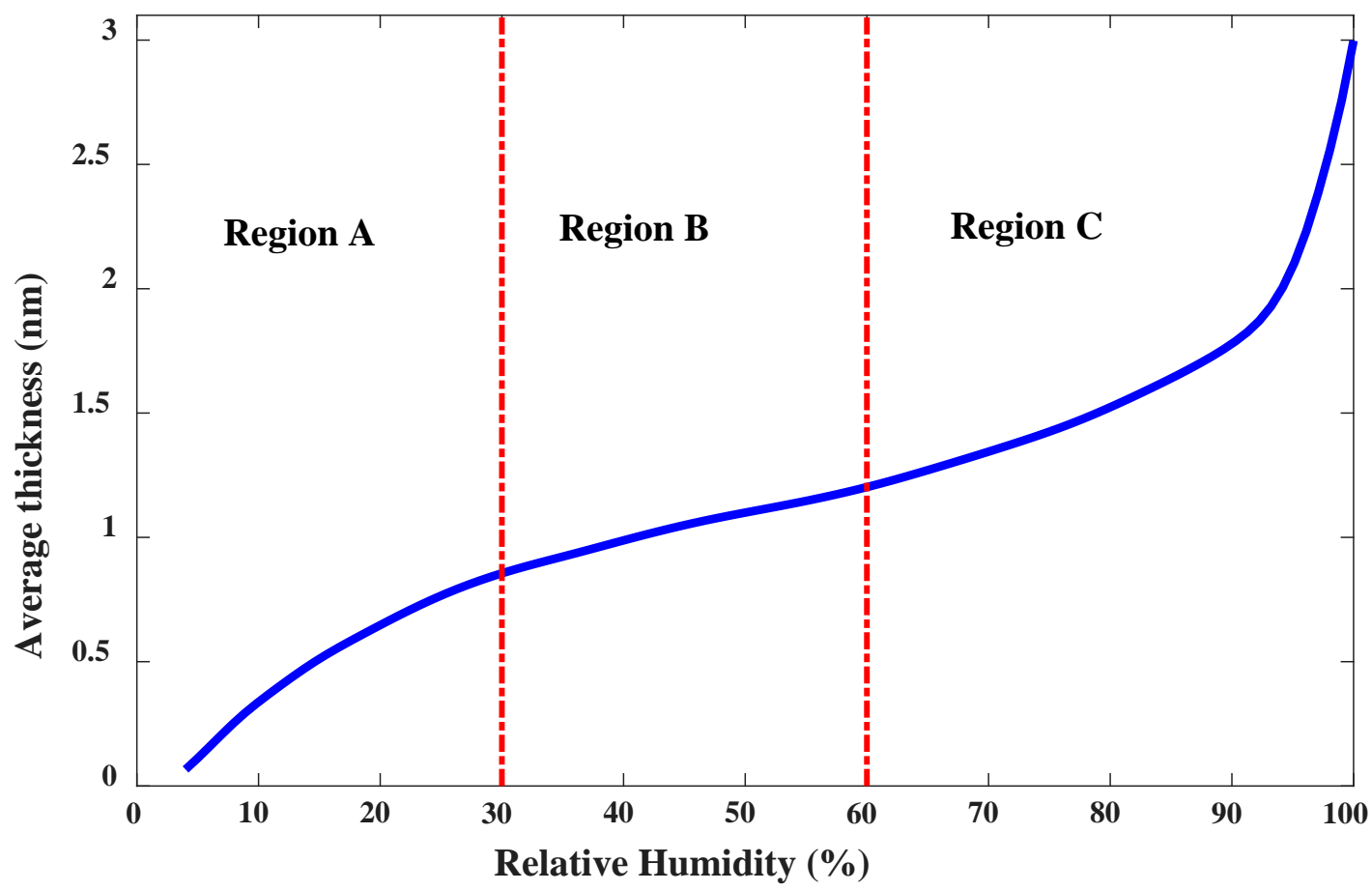

Fig. 7. Adsorption isotherm of water on a silicon-oxide surface (redrawn from Ref. [42]). 


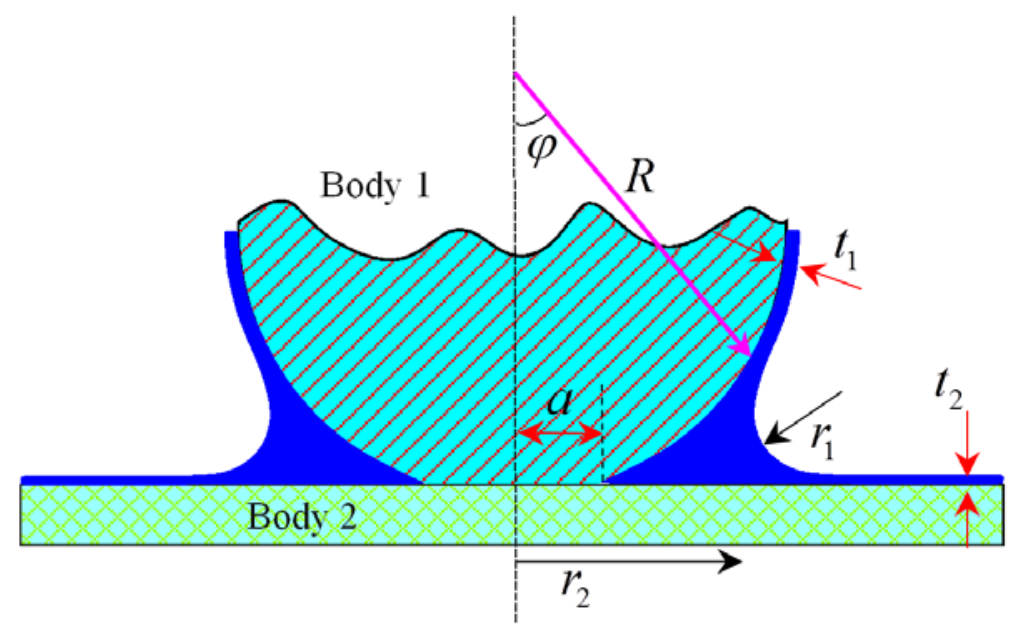

Fig. 8. Meniscus formation at a smooth ball-on-flat interface in the presence of an adsorbed water film. 

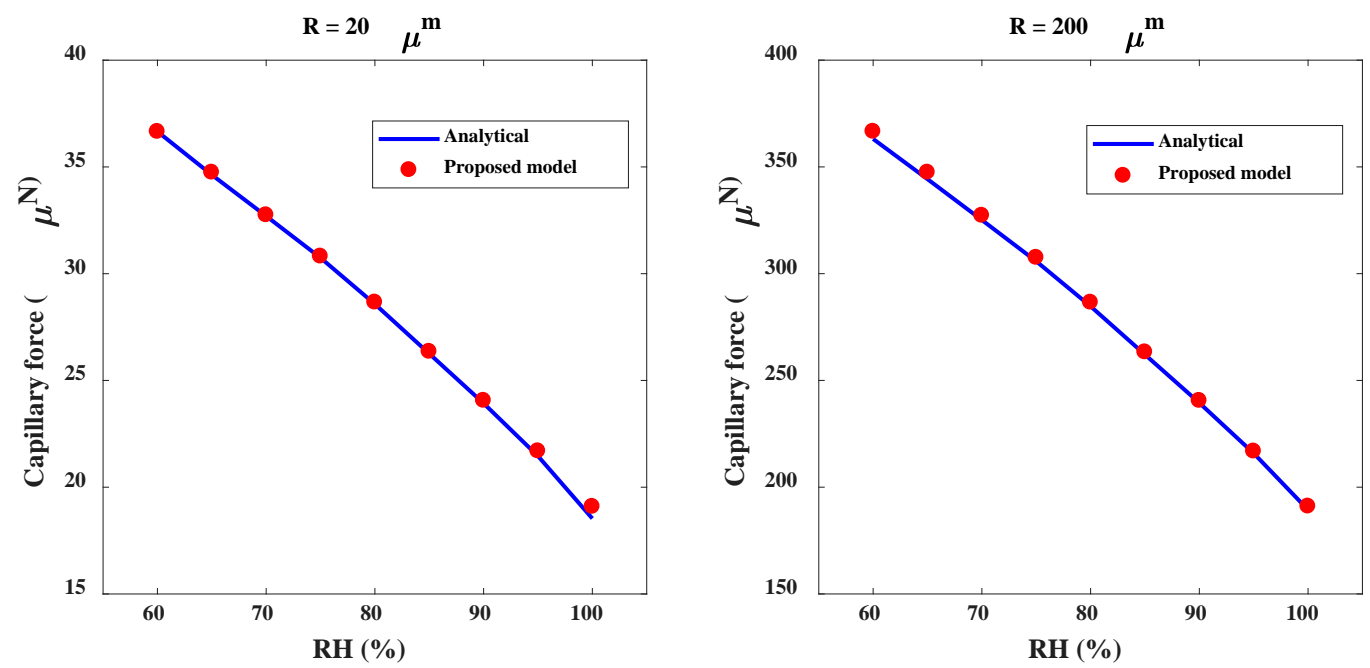

Fig. 9. Comparison of the analytical and numerical solutions for the capillary force at different RH for two different ball radii (R). 
(a) $\mathrm{L}_{\text {ac }} / \mathrm{L}=\mathbf{0 . 0 2}$

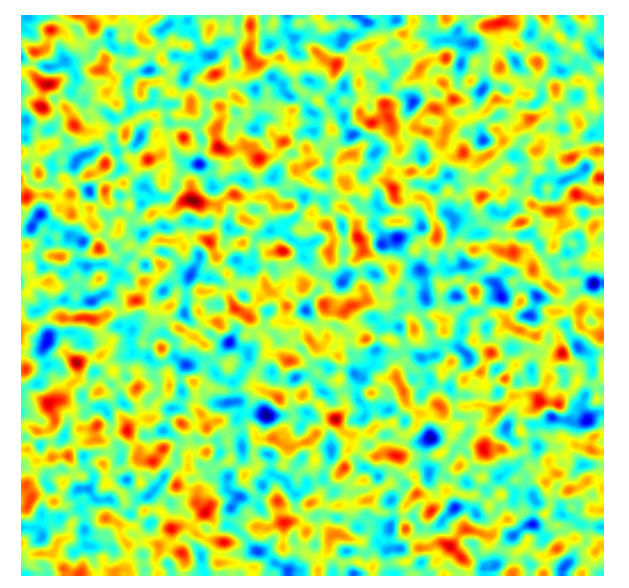

(b) $\mathrm{L}_{\text {ac }} / \mathrm{L}=\mathbf{0 . 0 5}$

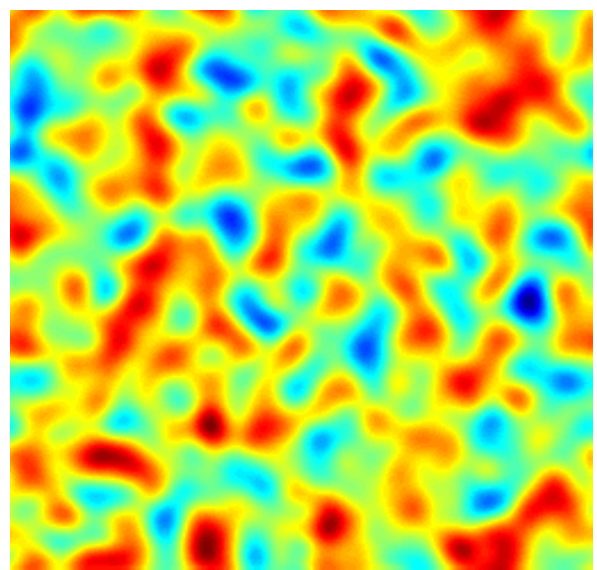

(c) $\mathrm{L}_{\text {ac }} / \mathrm{L}=\mathbf{0 . 1 0}$

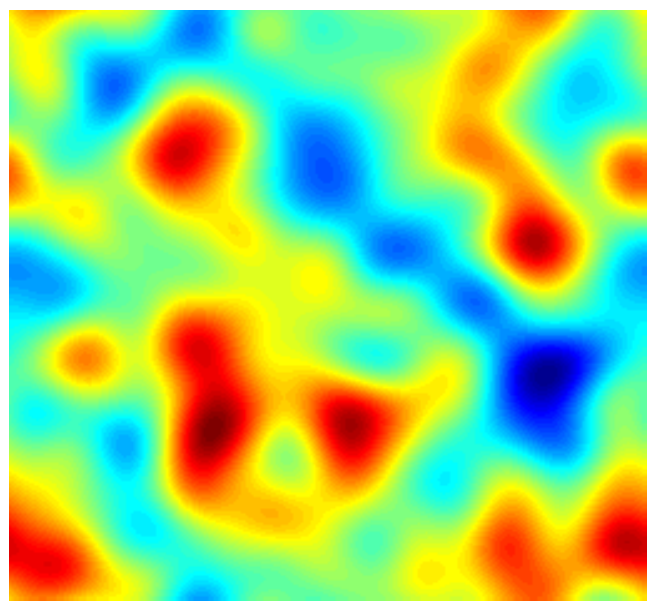

Fig. 10. The roughness height (in $\mathrm{nm}$ ) of the three generated Gaussian isotropic rough surfaces with different autocorrelation length ratio. 

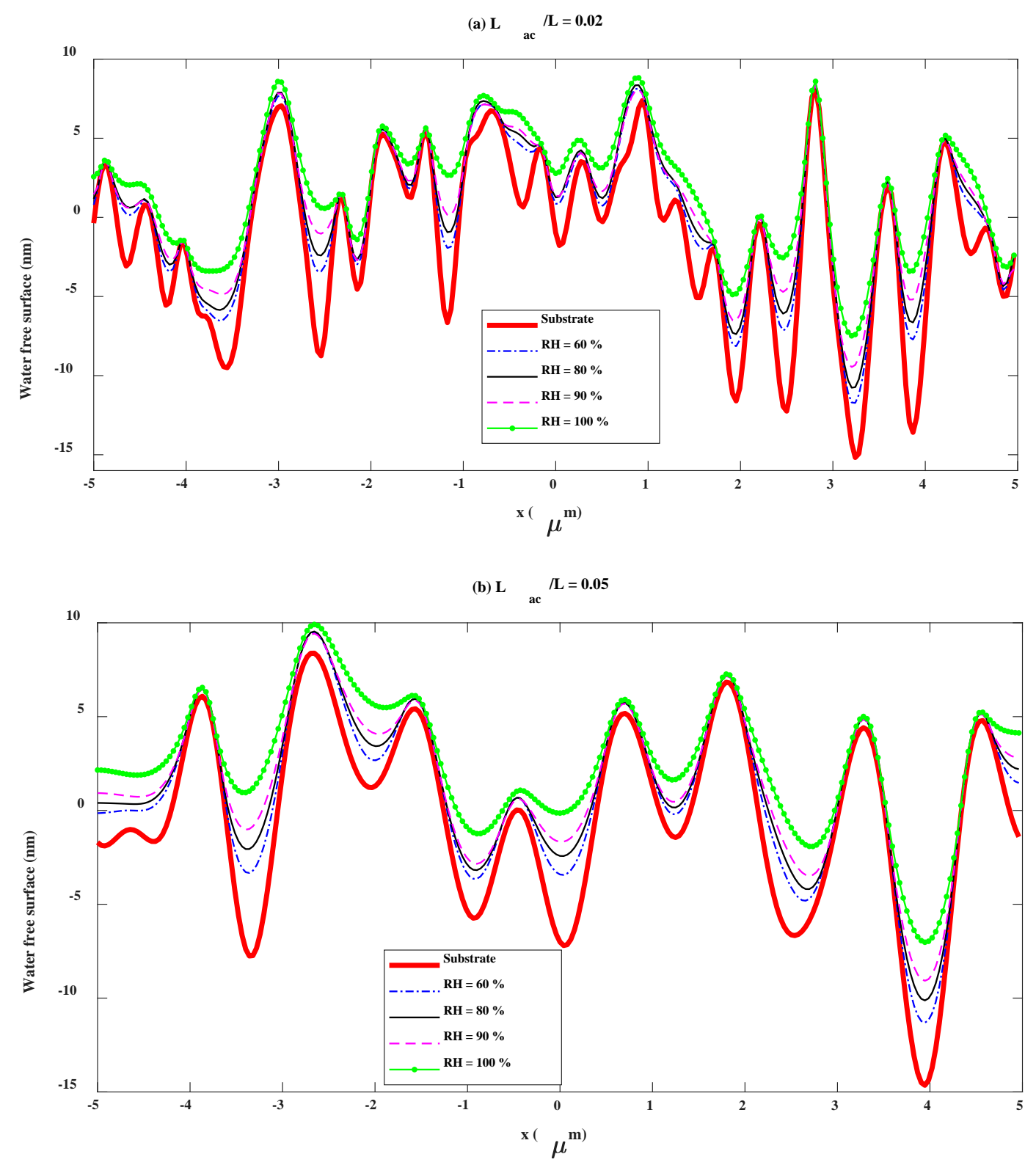
(c) $\mathrm{L} / \mathrm{L}=0.1$

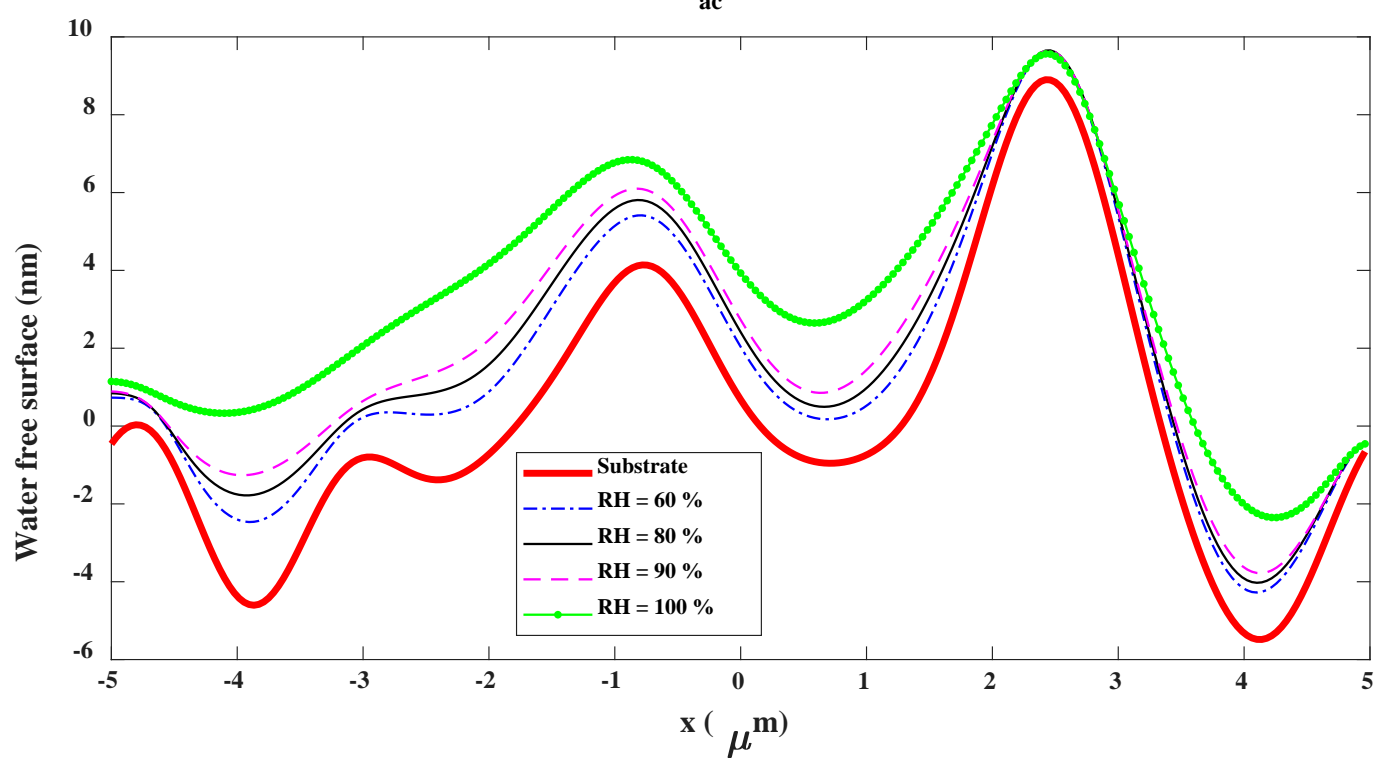

Fig. 11. The leveled water film on the rough surfaces at different RH. 
$\mathbf{R H}=\mathbf{6 0} \%$

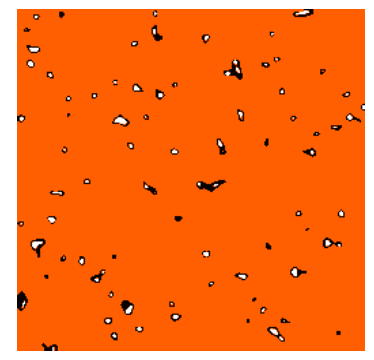




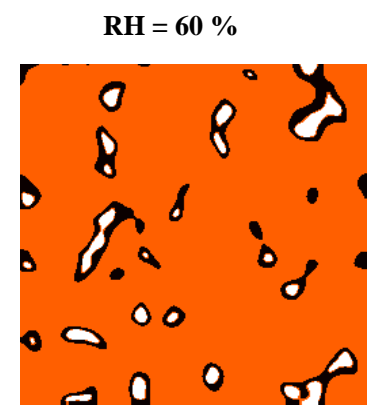




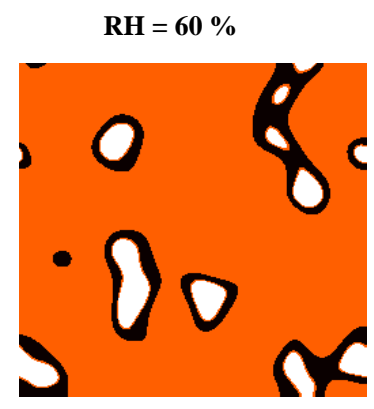

Fig. 12. The development of meniscus area with increasing RH at the three rough interfaces with different autocorrelation lengths. 
(a) $\mathrm{L}_{\text {ac }} / \mathrm{L}=\mathbf{0 . 0 2}$

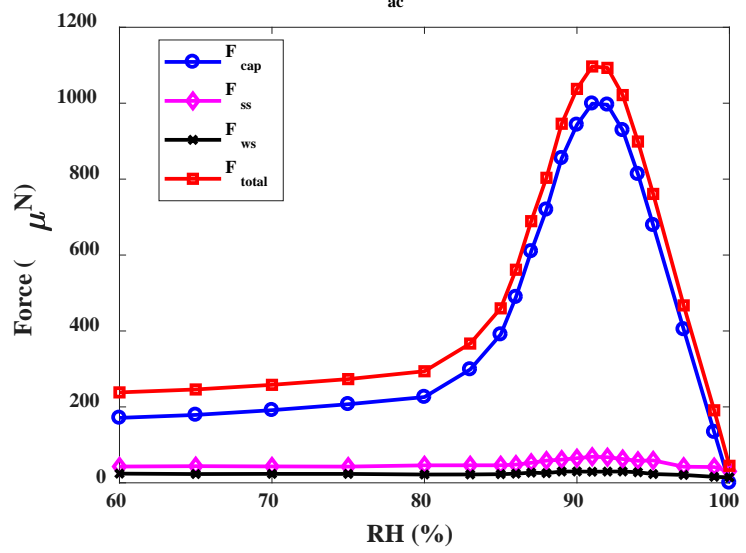

(c) $\mathrm{L} / \mathrm{L}=0.1$

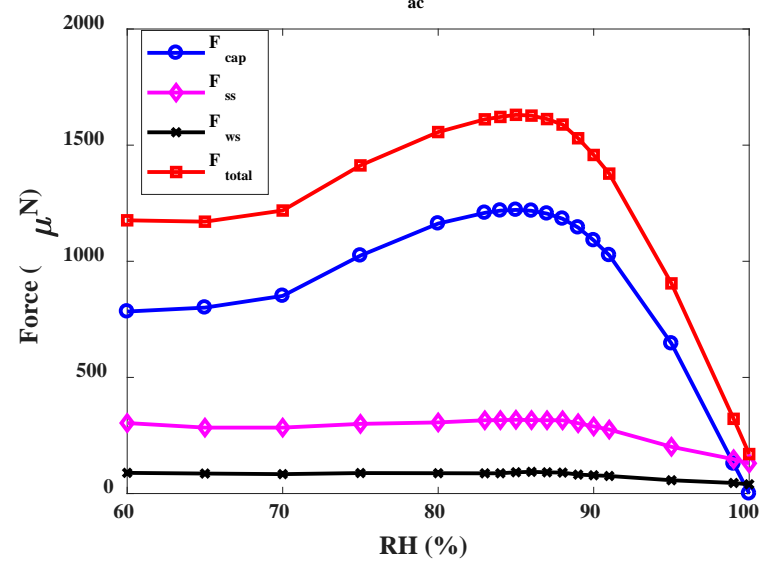

(b) $\mathrm{L}$ ac $/ \mathrm{L}=\mathbf{0 . 0 5}$
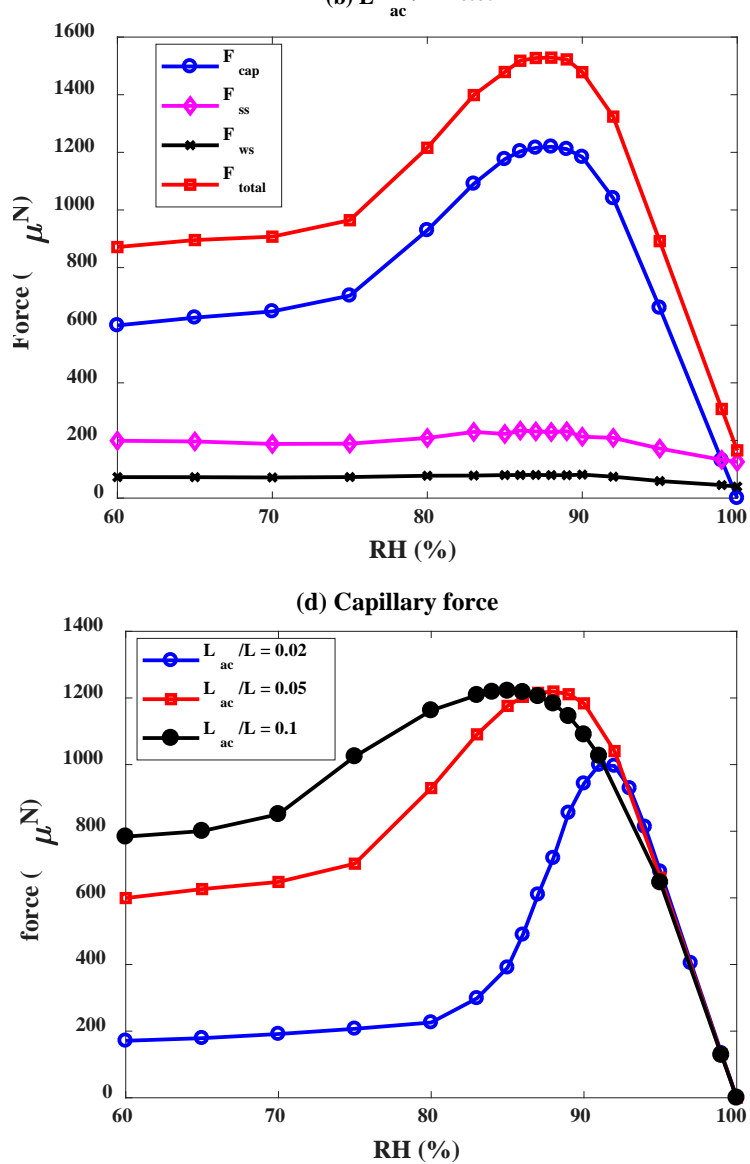

Fig. 13. Variation of different adhesive components vs. RH at three different auto-correlation lengths (a-c) and (d) comparison of the capillary force component. 


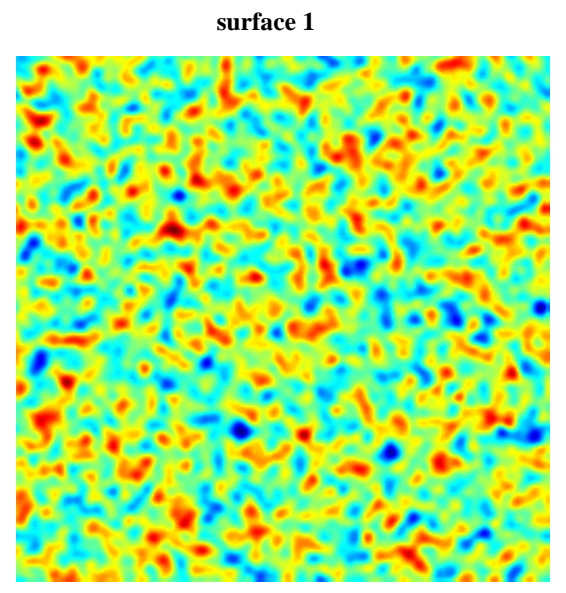

Fig. 14. Four different Gaussian rough surfaces with identical auto-correlation length ( $L_{a c} / L=0.02$ ), and RMS values ( $\sigma=5 \mathrm{~nm}$ ); surface 1 is the same as Fig. 10(a). 


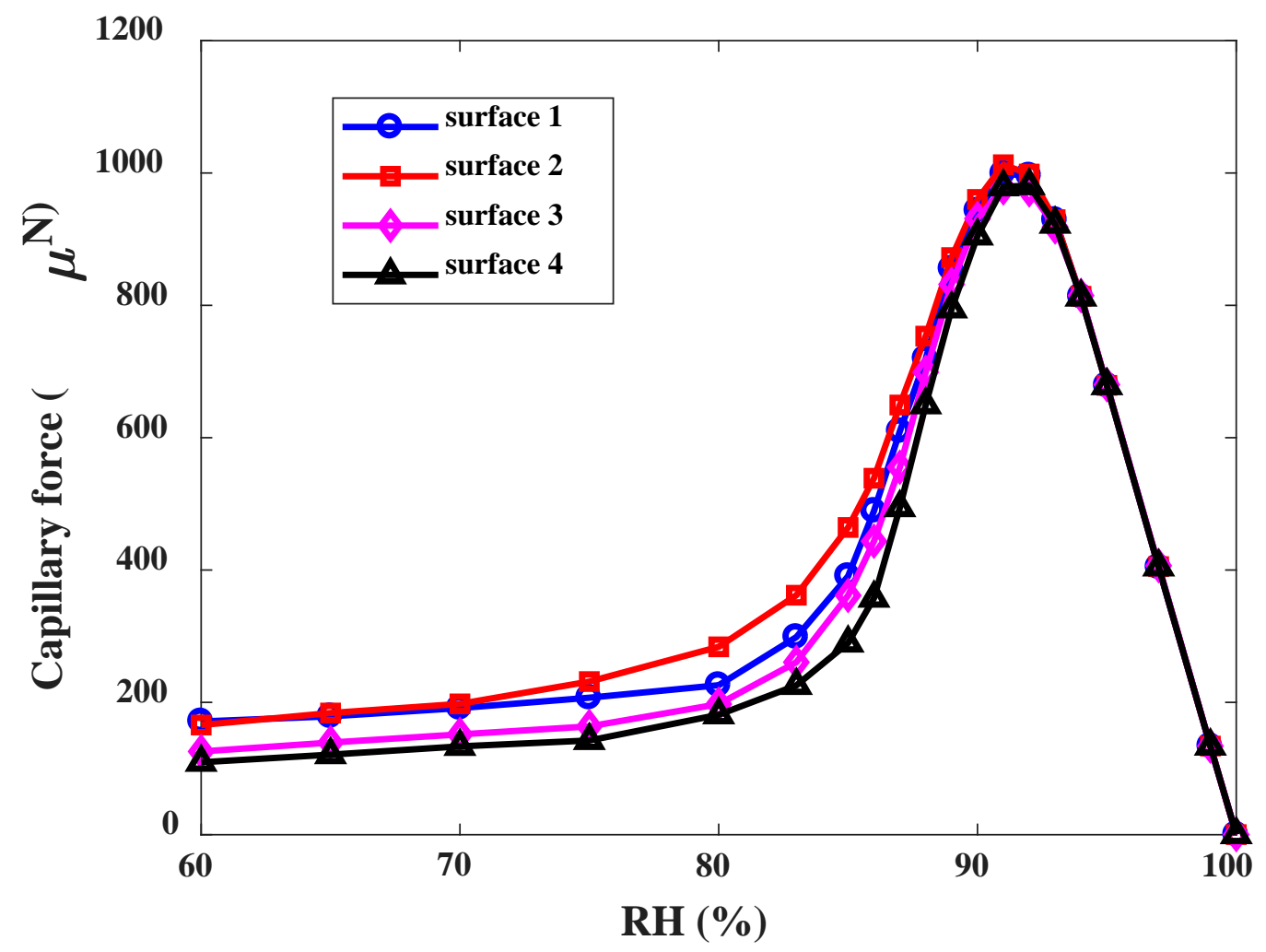

Fig. 15. The comparison of the capillary force for 4 different Gaussian rough surfaces with identical auto-correlation lengths and RMS values ( $\mathrm{L}_{\mathrm{ac}} / \mathrm{L}=0.02, \sigma=5 \mathrm{~nm}$ ). 


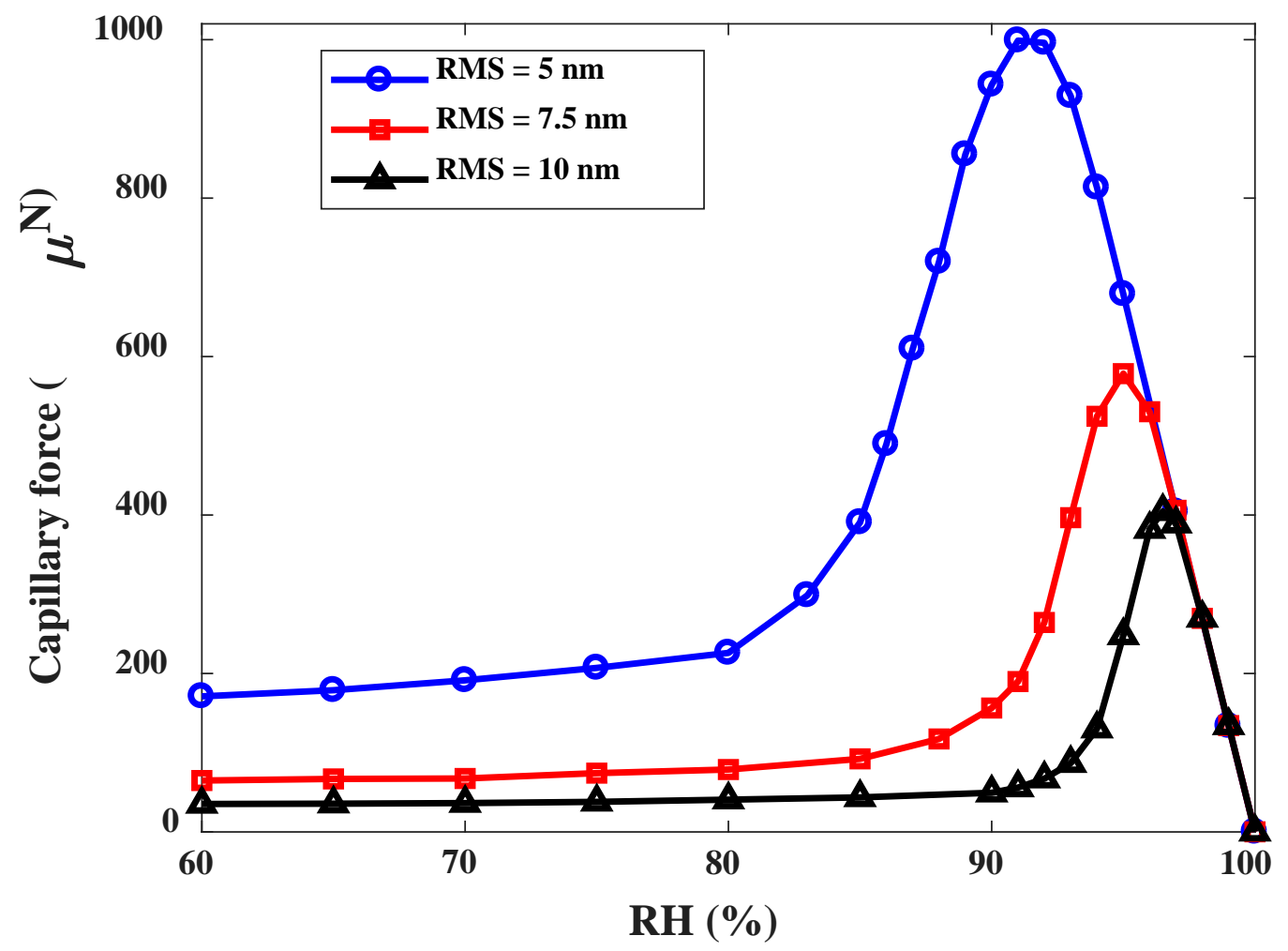

Fig. 16. The comparison of the capillary force for rough interface with different RMS values. 

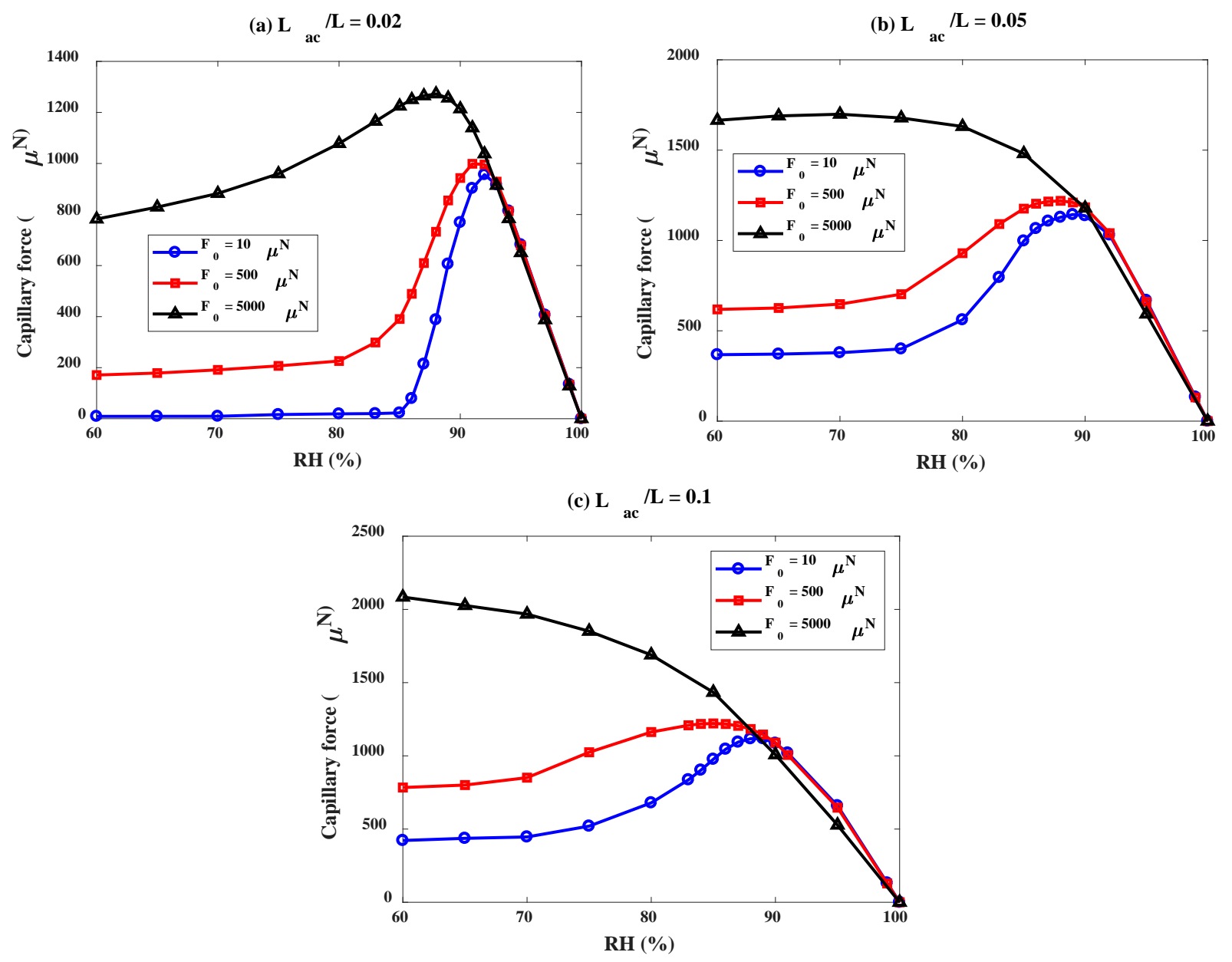

Fig. 17. The effect of external normal load on the capillary force. 

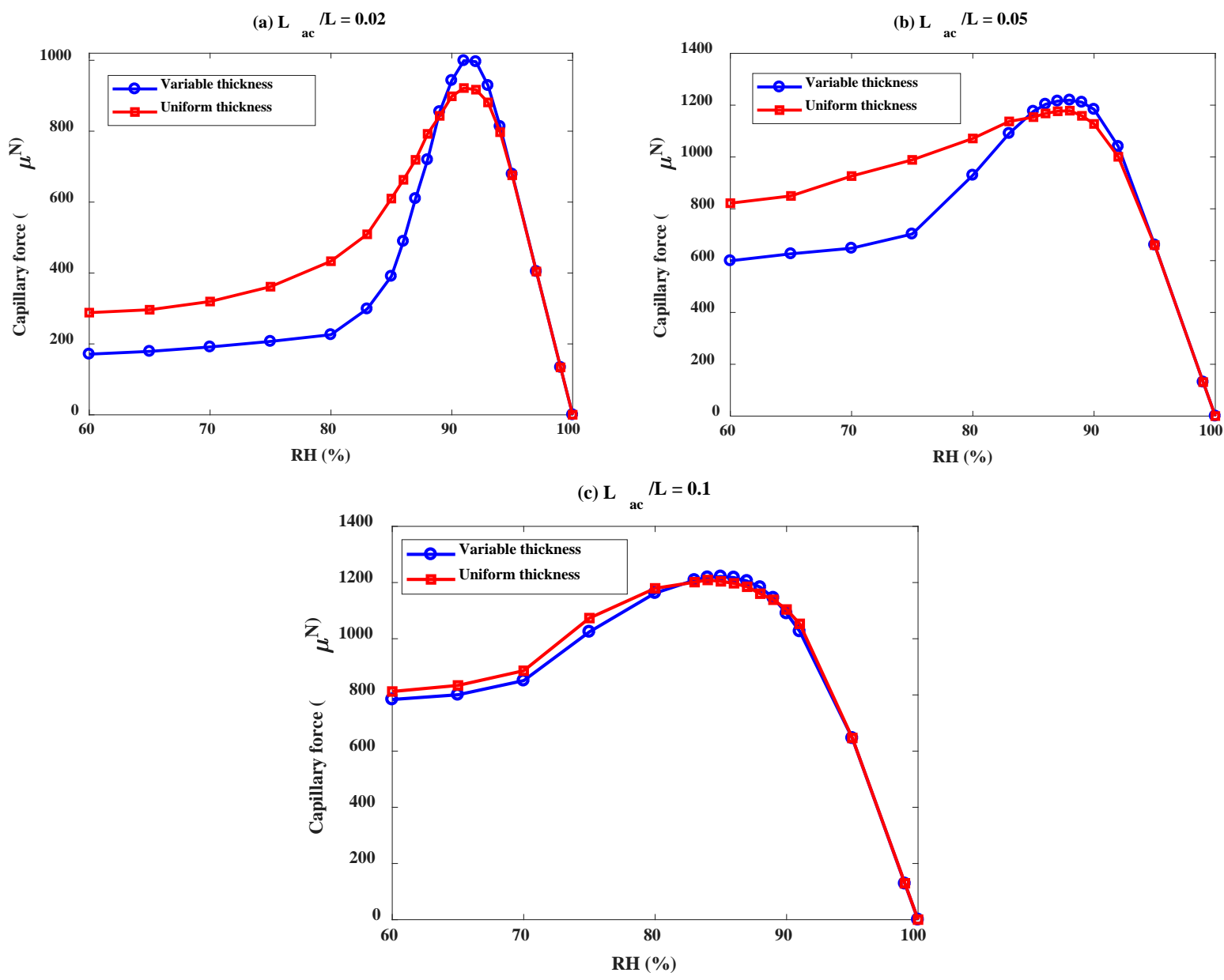

Fig. 18. The comparison of the capillary force with uniform and variable thickness of the water film over the rough surfaces. 